\title{
Heavy elements in old very metal-rich stars ${ }^{\star, \star \star}$
}

\author{
M. Trevisan ${ }^{1}$ and B. Barbuy ${ }^{2}$ \\ 1 Instituto Nacional de Pesquisas Espaciais/MCT, SP 12227-010 São José dos Campos, Brazil \\ e-mail: marina.trevisan@inpe.br \\ 2 Universidade de São Paulo, Rua do Matão 1226, 05508-900 São Paulo, Brazil \\ e-mail: b.barbuy@iag.usp.br \\ Received 1 November 2013 / Accepted 1 August 2014
}

\section{ABSTRACT}

\begin{abstract}
Context. We studied a sample of high proper motion, old and metal-rich dwarf stars, selected from the NLTT catalogue. The low pericentric distances and eccentric orbits of these solar neighbourhood stars indicate that they might have originated in the inner parts of the Galaxy.

Aims. Chemical tagging can probe the formation history of stellar populations. To identify the origin of a sample of 71 very metal-rich dwarf stars, we derive the abundances of the neutron-capture elements $\mathrm{Y}, \mathrm{Ba}, \mathrm{La}$, and $\mathrm{Eu}$.

Methods. The spectroscopic analysis is based on optical high-resolution échelle spectra obtained with the FEROS spectrograph at the ESO $1.52 \mathrm{~m}$ Telescope at La Silla, Chile. The abundances of Y, Ba, La, and Eu were derived through LTE analysis, employing the MARCS model atmospheres.

Results. The abundances of Y, Ba, $\mathrm{La}$, and Eu vs. Fe and $\mathrm{Mg}$ as the reference elements indicate similarities between our sample of old metal-rich dwarf stars and the thin disk. On the other hand, the abundance ratios using $\mathrm{O}$ as the reference element, as well as their kinematics, suggest that our sample is clearly distinct from the thin-disk stars. They could be old inner thin-disk stars, as suggested previously, or bulge stars. In either cases, they would have migrated from the inner parts of the Galaxy to the solar neighbourhood.
\end{abstract}

Key words. stars: abundances - stars: atmospheres - solar neighborhood

\section{Introduction}

The heavy elements Y, Ba, La, and Eu are among the most wellstudied neutron-capture elements because they show a number of measurable lines in stellar spectra. Chemical abundances in the solar system indicate that, while Y, Ba, and La, are dominantly formed through slow neutron-capture process, Eu is dominantly a rapid neutron-capture element. The s- and r-process contribution to the solar system abundances is given for example in studies by Seeger et al. (1965), Arlandini et al. (1999), Simmerer et al. (2004), and Sneden et al. (2008). At early times in the Galaxy, heavy elements are expected to be due to an r-process contribution, since there was no time for the main s-process to operate, as first suggested by Truran (1981). Recently, it was suggested by Cescutti \& Chiappini (2014) that s-process elements could have been produced by massive spinstars at early times in the halo. Chiappini et al. (2011) and Barbuy et al. (2014) also suggested to use abundances ratios of neutron-capture elements in old bulge stars to determine whether they are products of $\mathrm{r}$ - or s-process.

The sites of r-process element production are still not known (e.g. Schatz et al. 2001; Wanajo \& Ishimaru 2006; Kratz et al. 2007; Thielemann et al. 2011). Nucleosynthesis in neutron star mergers for the production of r-elements was favoured in recent works by Rosswog et al. (2014) and Wanajo et al. (2014). They confirmed that the so-called strong r-process yields elements with atomic number $\mathrm{A}>130$ (second peak of the heavy elements and above), whereas the so-called weak r-process could

^ Observations collected at the European Southern Observatory, La Silla, Chile

$\star \star$ Table 4 is only available in electronic form at the CDS via anonymous ftp to cdsarc.u-strasbg. fr (130.79.128.5) or via http://cdsarc.u-strasbg.fr/viz-bin/qcat?]/A+A/570/A22 take place in neutrino-driven winds of the same neutron star mergers and produce elements with $50<\mathrm{A}<130$, which includes the first peak of the heavy elements. Abundance ratios of first- and second-peak elements and s-dominant elements such as Ba over r-dominant elements such as Eu can contribute to distinguishing different nucleosynthesis process contributions (e.g. Siqueira-Mello et al. 2014; Siqueira-Mello \& Barbuy 2014).

In the seminal work by Käppeler et al. (1989) and Käppeler et al. (2011) three different channels for the production s-process elements are described: the weak s-process in He-burning and C-burning shells of massive stars, and the main and the strong s-process taking place in asymptotic giant branch (AGB) stars. These processes were inspected in detail by Gallino et al. (1998) and Travaglio et al. (2004), among others.

Heavy-element abundances have been examined more frequently in metal-poor than in metal-rich stars. Previous heavyelement abundance derivations in metal-rich stars were carried out for thin-disk (Edvardsson et al. 1993; Reddy et al. 2003; Allende Prieto et al. 2004), and thick-disk stars (Reddy et al. 2006; Bensby et al. 2005). Recently, Mishenina et al. (2013) derived heavy-element abundances of thin-, thick-disk and Hercules-stream stars, and abundances of bulge-field stars were determined by Bensby et al. (2013) and Johnson et al. (2012, 2013). In addition, heavy-element abundances were derived for open-cluster stars (e.g. Jacobson \& Friel 2013) and for two bulge barium stars by Lebzelter et al. (2013).

We here derive Y, Ba, La, and Eu abundances in 71 metalrich stars in the solar neighbourhood. The sample stars are high proper motion, old stars, with low maximum heights above the Galactic plane, and their kinematics is indicative of stars wandering from inside the Galactic bar (Raboud et al. 1998). Trevisan et al. (2011, hereafter Paper I) presented the detailed analysis of the sample and derived their $\alpha$-element abundances. 
Based on their kinematical properties, in Paper I we identified the present sample as partly belonging to the thin disk (11 stars), a majority of them to the thick disk (42 stars), and 17 stars to an intermediate population. The thick-disk and intermediate populations might be similar to the thickdisk-kinematics-thin-disk-abundances (designated TKTA) stars (Reddy et al. 2006), which show thick-disk kinematics combined with thin-disk abundances.

To probe the formation history of this sample, in Paper I we derived their metallicities, $\alpha$-element abundances $(\mathrm{O}, \mathrm{Mg}$, $\mathrm{Si}, \mathrm{Ca}$, and $\mathrm{Ti}$ ), ages, and Galactic orbits. We showed that models of radial mixing and dynamical effects of the bar and bar/spiral arms might explain the presence of these old metalrich dwarf stars in the solar neighbourhood. In Paper I we concluded that our sample stars might be identified as old thin-disk stars (Haywood 2008). To better understand the properties of these stars, we here study the abundances of the neutron-capture elements Y, Ba, La, and Eu.

The paper is organized as follows: in Sect. 2, we briefly describe the sample and the derivation of stellar parameters that were presented in Paper I. The Y, Ba, La, and Eu abundance derivation is described in Sect. 3, and the results are presented and discussed in Sects. 4 and 5. Finally, the results are summarized in Sect. 6.

\section{Sample}

The sample star selection, their kinematics, the stellar parameter determination, and the abundance analysis of $\alpha$-elements are described in detail in Paper I. Below we summarize the information about the sample stars.

The sample comprises high proper motion and metal-rich dwarf stars from the NLTT catalogue (Luyten 1980), as described in Grenon (1999) and Paper I. The observations were carried out in 2000-2002, within an ESO-ON-IAG agreement. The spectra were obtained using the Fiber-Fed Extended Range Optical Spectrograph (FEROS; Kaufer et al. 2000) at the $1.52 \mathrm{~m}$ telescope at ESO, La Silla. The total wavelength coverage is 3560-9200 $\AA$ with a resolving power $(R=\lambda / \Delta \lambda)=48000$, and with mean signal-to-noise ratios of $\sim 100$.

\subsection{Galactic orbits and membership assignment}

Grenon (1999) derived $U, V$, and $W$ space velocities for the sample stars, which were used to calculate the Galactic orbits. The GRINTON integrator was used for this purpose (Carraro et al. 2002; Bedin et al. 2006). This code integrates the orbits back in time for several Galactic revolutions and returns the minimum and maximum distances from the Galactic centre $\left(R_{\min }, R_{\max }\right)$, maximum height from the Galactic plane $\left(Z_{\max }\right)$ and the eccentricity $e$ of the orbit.

Based on Galactic velocities $U, V, W$ and eccentricities $e$, we estimated in Paper I the probability of each sample star to belong to either the thin or the thick disk. The procedure adopted relies on the assumption that the space velocities of each population (thin disk, thick disk and halo) follow a Gaussian distribution, with given mean values and dispersions $\sigma_{U}, \sigma_{V}, \sigma_{W}$. We used the velocity ellipsoids by Soubiran et al. (2003) and found that 42 stars in the sample can be assigned to the thick disk, 11 are more likely to be thin-disk stars, and the other 17 stars are intermediate between thin- and thick-disk components.

\subsection{Stellar parameters}

The effective temperatures $T_{\text {eff }}$ were calculated from the $V-$ $K_{\mathrm{S}}$ colour using the Casagrande et al. (2010) colour-temperature relations. The surface gravities $\log g$ were derived using the HIPPARCos parallaxes, and the stellar ages and masses from the Yonsei-Yale evolutionary tracks ( $Y^{2}$ Demarque et al. 2004). Then, fixing $T_{\text {eff }}$ and $\log g$, the iron abundances were derived from $\mathrm{Fe}$ I and $\mathrm{Fe}$ II lines through a local thermodynamic equilibrium (LTE) analysis, employing plane-parallel MARCS model atmospheres (Gustafsson et al. 2008) with scaled-solar composition. The microturbulence velocity, $v_{\mathrm{t}}$, was obtained by imposing constant iron abundance as a function of equivalent width. For our cooler stars $\left(T_{\text {eff }}<5000 \mathrm{~K}\right)$, a positive trend of $[\mathrm{Fe} / \mathrm{H}]$ vs. equivalent width was found even when $v_{\mathrm{t}} \sim 0 \mathrm{~km} \mathrm{~s}^{-1}$. For this reason, we considered that $v_{\mathrm{t}}$ can be defined as a function of temperature and gravity, and using stars with $T_{\text {eff }}>5200 \mathrm{~K}$, we defined $v_{\mathrm{t}}=f\left(T_{\mathrm{eff}}, \log g\right)$ and then extrapolated this function to $T_{\text {eff }}<5200 \mathrm{~K}$. We adopted $0.3 \mathrm{~km} \mathrm{~s}^{-1}$ from the extrapolation of our fit. The metallicity was then replaced by the new iron abundance, and the procedure was repeated until there were no significant changes in $\left(T_{\text {eff }}, \log g,[\mathrm{Fe} / \mathrm{H}]^{1}\right)$.

The stellar parameters were tested against excitation and ionization equilibria, and $T_{\text {eff }}$ and $\log g$ were further adjusted when necessary. Only two stars in the sample (HD 94374 and HD 182572) required adjustments to be in satisfactory spectroscopic equilibrium.

The final adopted temperatures, gravities, and metallicities were compared with data available in the literature. For comparison purposes, the parameters $\left(T_{\mathrm{eff}}, \log g,[\mathrm{Fe} / \mathrm{H}]\right)$ were retrieved from the PASTEL catalogue (Soubiran et al. 2010), which compiles stellar atmospheric parameters obtained from the analysis of high-resolution, high signal-to-noise spectra. The parameters of 38 of our sample stars are available in this catalogue. Differences between temperatures considered here and those from the PASTEL catalogue do not exceed 2\%, except for two stars (HD 31827 and HD 35854). Gravities and metallicities also agree well, with differences within $\sim 0.2$ dex.

\section{Abundance derivation}

We derived abundances of yttrium, barium, lanthanum, and europium for the sample stars. We performed a spectral synthesis, and the abundances were obtained by minimising the $\chi^{2}$ between the observed and synthetic spectra. The synthetic spectra were obtained using the PFANT code described in Cayrel et al. (1991), Barbuy et al. (2003), and Coelho et al. (2005). The MARCS model atmospheres (Gustafsson et al. 2008) were employed.

The solar abundances from the literature and adopted values are reported in Table 1 . The most updated values computed with 3D model atmospheres by Asplund et al. (2009) and Grevesse et al. (2014) recommended values were not adopted here because we used 1D model atmospheres to derive the abundances of the sample stars.

All lines were checked against the solar spectrum obtained with the same instrumentation as the program stars, and $g f$-values were adjusted when necessary. For lines of the elements Ba II, La II, and Eu II, the hyperfine structure (HFS) was taken into account, as described in the following sections.

\footnotetext{
1 We use the standard notation, i.e., for elements $\mathrm{A}$ and $\mathrm{B},[\mathrm{A} / \mathrm{B}]=$ $\log \left(N_{\mathrm{A}} / N_{\mathrm{B}}\right)_{\text {star }}-\log \left(N_{\mathrm{A}} / N_{\mathrm{B}}\right)_{\odot}$ and $\epsilon(\mathrm{A})=\log \left(N_{\mathrm{A}} / N_{\mathrm{H}}\right)+12$.
} 

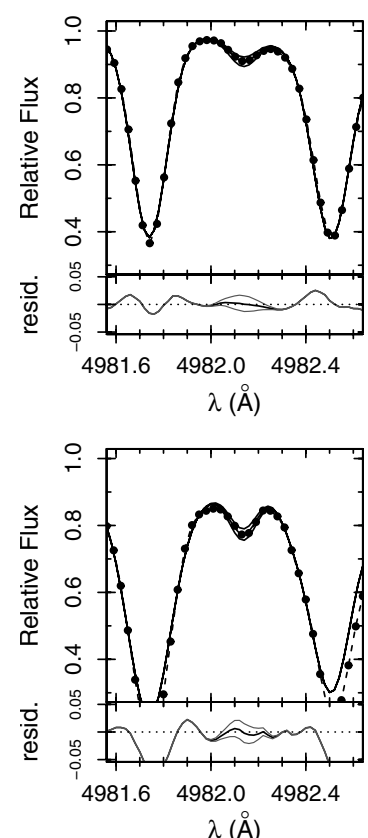

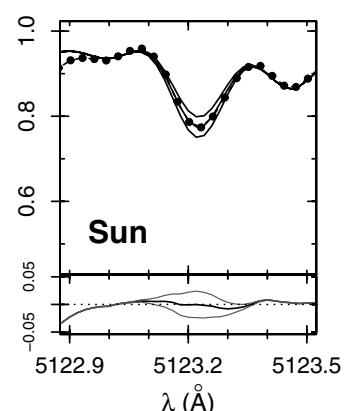

$\lambda(\AA)$

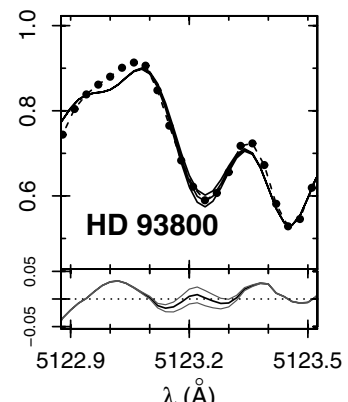

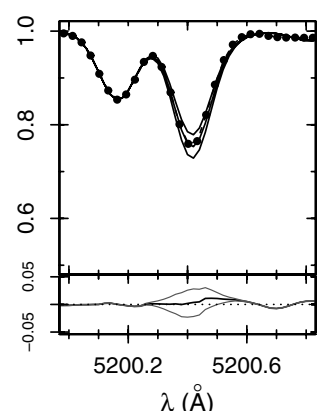

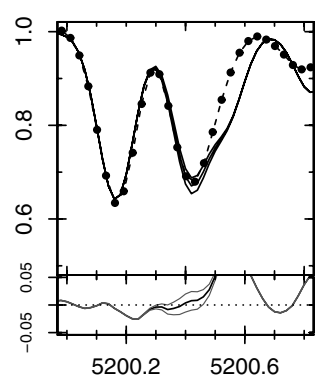

$\lambda(\AA)$

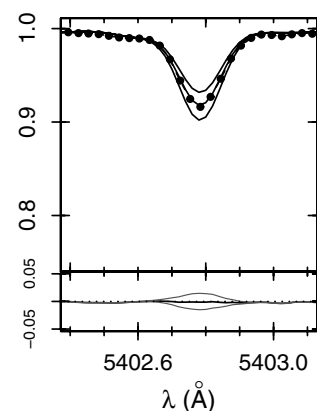

$\lambda(\AA)$

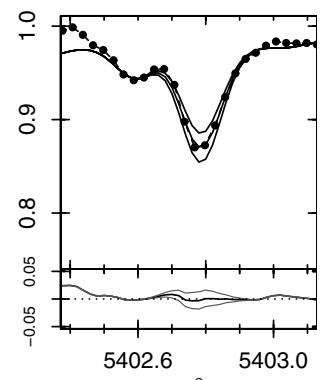

$\lambda(\AA)$

Fig. 1. Synthetic and observed profiles of Y II lines. Upper panels: solar spectrum in the region of Y II lines. Solid lines indicate the synthetic spectra for $\epsilon(\mathrm{Y})=2.14,2.24,2.34$; the dots indicate the observed spectrum. Lower panels: spectral synthesis of Y II lines for HD 93800.

Table 1. Solar $r$ - and $s$-process fractions (Simmerer et al. 2004) and solar abundances from 1: Grevesse \& Sauval (1998); 2: Asplund et al. (2009); 3: Lodders et al. (2009); 4: Grevesse et al. (2014).

\begin{tabular}{ccccccccc}
\hline \hline \multirow{2}{*}{ El. } & \multirow{2}{*}{$Z$} & \multicolumn{2}{c}{ Fraction } & \multicolumn{5}{c}{$\epsilon(X)_{\odot}$} \\
\cline { 5 - 9 } & & $r$ & $s$ & $(1)$ & $(2)$ & $(3)$ & $(4)$ & $(5)$ \\
\hline $\mathrm{Fe}$ & 26 & - & - & 7.50 & 7.50 & 7.45 & 7.45 & 7.50 \\
$\mathrm{Y}$ & 39 & 0.281 & 0.719 & 2.24 & 2.21 & 2.21 & 2.21 & 2.24 \\
$\mathrm{Ba}$ & 56 & 0.147 & 0.853 & 2.13 & 2.18 & 2.18 & 2.25 & 2.13 \\
$\mathrm{La}$ & 57 & 0.246 & 0.754 & 1.17 & 1.10 & 1.14 & 1.11 & 1.22 \\
$\mathrm{Eu}$ & 63 & 0.973 & 0.027 & 0.51 & 0.52 & 0.52 & 0.52 & 0.51 \\
\hline
\end{tabular}

Notes. Adopted abundances are given in Col. 5.

\section{Yttrium}

The Y II lines were selected from Hannaford et al. (1982), Spite et al. (1987), Hill et al. (2002), and Grevesse et al. (2014), and they are listed in Table 2. The $\log g f$ values were adjusted to fit the solar spectrum. Figure 1 presents the solar observed and synthetic spectra in the region of Y II lines. The spectrum synthesis fitting of Y II lines to the observed profiles for one of the sample stars, HD 93800, is also presented in Fig. 1. The yttrium line list comprises lines that systematically give reliable abundances, as shown in Fig. 2. Given that $\langle A\rangle_{i}$ is the yttrium abundance of each star and $A_{\lambda i}$ is the abundance derived from an individual line, the quantity $A_{\lambda i}-\langle A\rangle_{i}$ indicates the departure of the abundance derived from the line $i$. This approach allows us to detect lines that give abundances systematically higher or lower than the average abundance of the star and lines that seem to lead to inaccurate abundance values. The abundances $A_{\lambda i}$ presented in Fig. 2 correspond to the corrected values obtained by removing the dependence with temperature (see Sect. 3.1).

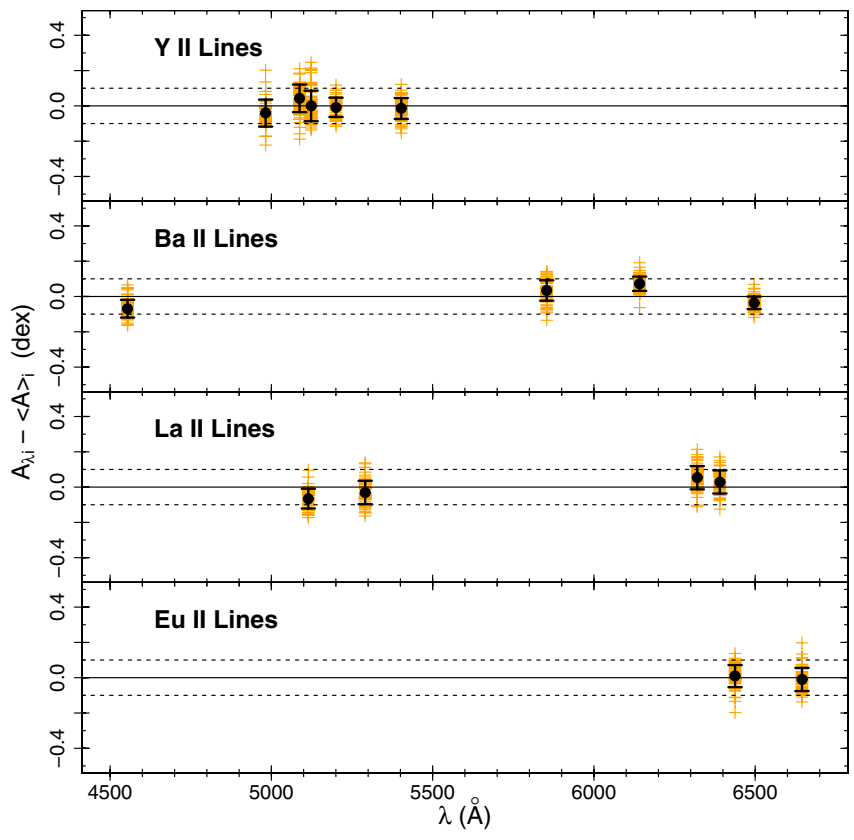

Fig. 2. Selected $\mathrm{Y}, \mathrm{Ba}, \mathrm{La}$, and Eu lines. The abundance of each star, $\langle A\rangle_{i}$, is the average of abundances derived from individual lines, $A_{\lambda i}$. Therefore, $A_{\lambda i}-\langle A\rangle_{i}$ indicates the differences between the mean abundance value and those derived from individual lines. The mean deviation for each line is represented by black circles. Dashed lines indicate $\pm 0.1 \mathrm{dex}$.

\section{Barium}

The barium abundances were obtained using Ba II lines at 4554, 5853, 6141, and $6496 \AA$, as listed in Table 2. Odd-numbered Ba isotopes exhibit hyperfine splitting, which was taken into account by employing a code made available by McWilliam (1998), following the calculations described by Prochaska \& McWilliam (2000), as reported in Barbuy et al. (2014). We 
Table 2. Line list.

\begin{tabular}{cccccccccccc}
\hline \hline$\lambda(\AA)$ & Species & $\begin{array}{c}\chi_{\mathrm{exc}} \\
\mathrm{(eV)}\end{array}$ & $\begin{array}{c}\log g f \\
\text { (adopted) }\end{array}$ & $\begin{array}{c}\log g f \\
\text { (HPC+02) }\end{array}$ & $\begin{array}{c}\log g f \\
\text { (SHFS87) }\end{array}$ & $\begin{array}{c}\log g f \\
(\text { HLG+82) }\end{array}$ & $\begin{array}{c}\log g f \\
\text { (GSAS14) }\end{array}$ & $\begin{array}{c}\log g f \\
\text { (NIST) }\end{array}$ & $\begin{array}{c}\log g f \\
\text { (Kurucz) }\end{array}$ & $\begin{array}{c}\log g f \\
\text { (VALD) }\end{array}$ & $\begin{array}{c}\log g f \\
(\mathrm{MPK} 13)\end{array}$ \\
\hline 4982.14 & Y II & 1.03 & -1.34 & - & -1.26 & -1.29 & - & - & -1.29 & -1.29 & -1.26 \\
5087.43 & Y II & 1.08 & -0.38 & -0.17 & -0.20 & -0.17 & -0.17 & - & -0.17 & -0.17 & -0.26 \\
5123.21 & Y II & 0.99 & -0.83 & -0.83 & - & -0.83 & - & - & -0.83 & -0.83 & - \\
5200.41 & Y II & 0.99 & -0.62 & -0.57 & - & -0.57 & -0.57 & - & -0.57 & -0.57 & -0.63 \\
5402.78 & Y II & 1.84 & -0.58 & - & -0.59 & - & - & - & -0.51 & -0.51 & -0.55 \\
4554.03 & Ba II & 0.00 & $+0.30^{a}$ & +0.17 & - & - & +0.17 & +0.14 & +0.17 & +0.17 & - \\
5853.69 & Ba II & 0.60 & $-0.90^{a}$ & -1.01 & -0.53 & - & -1.03 & -0.91 & -1.00 & -1.00 & - \\
6141.71 & Ba II & 0.70 & $+0.15^{a}$ & -0.07 & +0.40 & - & - & -0.03 & -0.08 & -0.08 & - \\
6496.91 & Ba II & 0.60 & $-0.10^{a}$ & - & +0.18 & - & -0.41 & -0.41 & -0.38 & -0.38 & - \\
5114.55 & La II & 0.24 & $-1.02^{a}$ & - & - & - & - & - & -1.06 & -1.03 & - \\
5290.82 & La II & 0.00 & $-1.70^{a}$ & - & -1.29 & - & - & - & -1.75 & -1.65 & - \\
6320.38 & La II & 0.17 & $-1.32^{a}$ & -1.52 & -1.43 & - & - & - & -1.61 & -1.56 & -1.33 \\
6390.48 & La II & 0.32 & $-1.31^{a}$ & - & - & - & - & - & -1.45 & -1.41 & - \\
6437.64 & Eu II & 1.32 & $+0.26^{a}$ & -0.32 & - & - & - & - & -0.28 & -0.32 & - \\
6645.11 & Eu II & 1.38 & $+0.20^{a}$ & +0.12 & - & - & - & - & +0.20 & +0.12 & - \\
\hline
\end{tabular}

Notes. HPC+02: Hill et al. (2002); SHFS87: Spite et al. (1987); HLG+82: Hannaford et al. (1982); GSAS14: Grevesse et al. (2014); MPK13: Mishenina et al. (2013). ${ }^{(a)} \log g f$ reported corresponds to the sum of individual values.
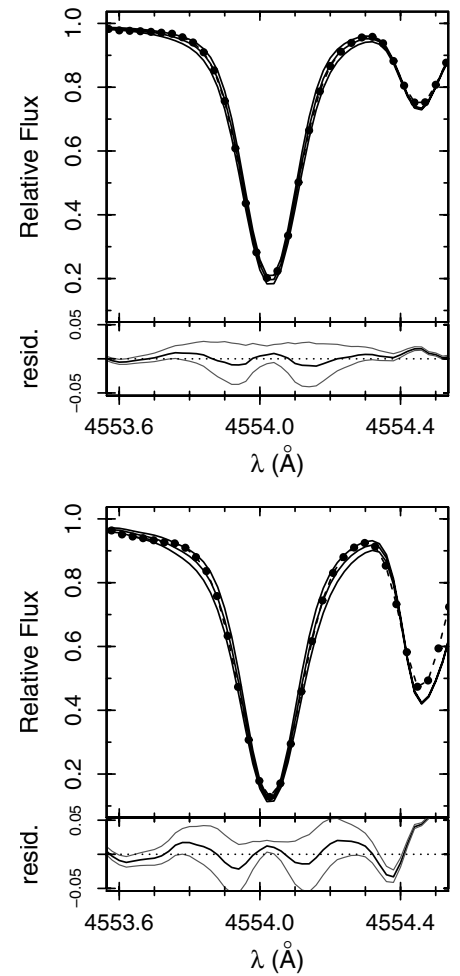
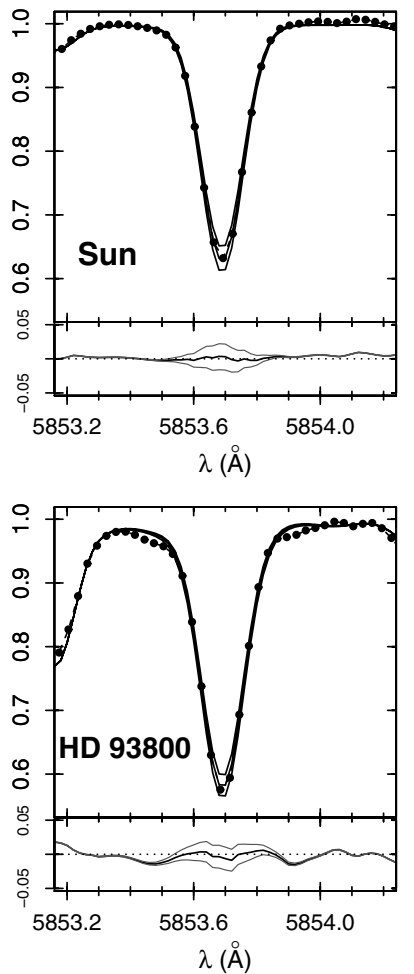
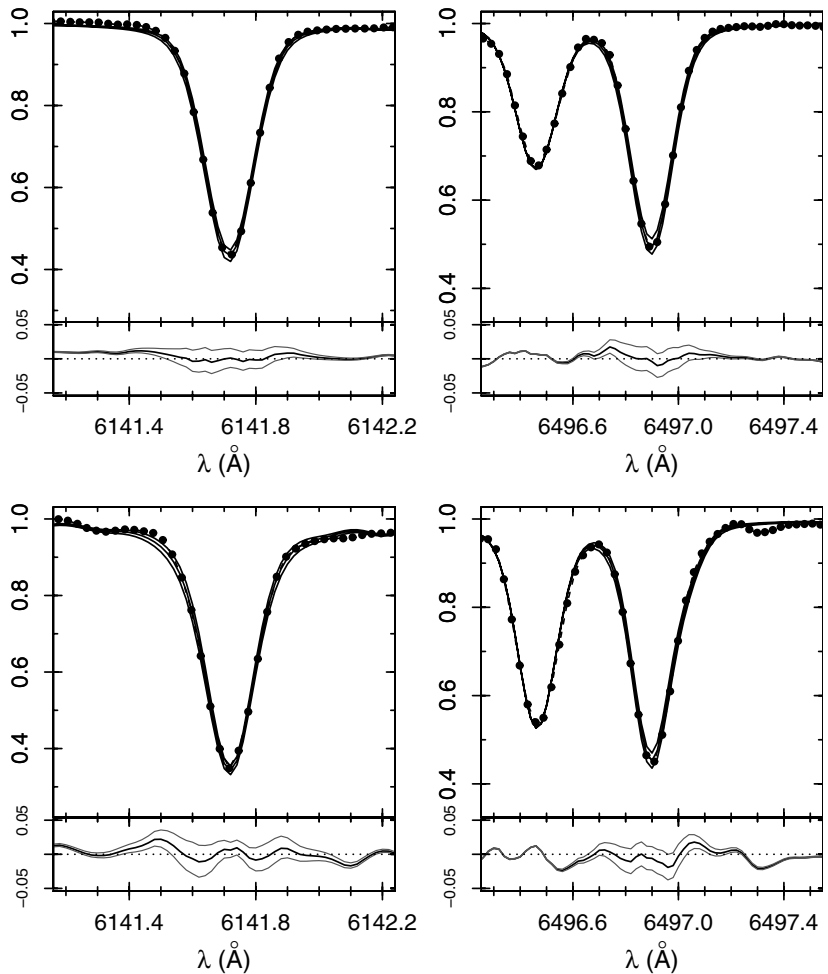

Fig. 3. Spectra in the region of Ba II lines. Upper panels: solar spectrum. Solid lines indicate the synthetic spectra for $\epsilon(\mathrm{Ba})=2.03,2.13,2.23$; the observed spectrum is represented by dots. Lower panels: spectrum synthesis fitting of Ba II lines for HD 93800.

considered that the nuclides ${ }^{134} \mathrm{Ba},{ }^{135} \mathrm{Ba},{ }^{136} \mathrm{Ba},{ }^{137} \mathrm{Ba},{ }^{138} \mathrm{Ba}$ contribute $2.42 \%, 6.59 \%, 7.85 \%, 11.23 \%$, and $71.7 \%$ to the total abundance, respectively (Lodders et al. 2009). Figure 3 shows the spectra of the Sun and the star HD 93800 in the region of Ba II lines. To fit the solar spectrum, the total $\log g f$ of the $\mathrm{Ba}$ lines was increased by $\sim 0.1-0.2$ dex with respect to values from the literature. The source of this difference is the adopted value for the solar microturbulence velocity; we used the same value as in Paper I $\left(v_{\mathrm{t}, \odot}=0.90 \mathrm{~km} \mathrm{~s}^{-1}\right)$. We checked the reliability of abundances derived from individual $\mathrm{Ba}$ lines using the same procedure as applied for Y lines, as presented in Fig. 2. The mean deviation of abundances derived from each $\mathrm{Ba}$ line do not exceed 0.1 dex.

\section{Lanthanum}

The La abundances were obtained using the lines listed in Table 2. The solar and HD 15133 spectra in the region of La lines are shown in Fig. 4. The HFS of the La II line at $5114 \AA$ was 

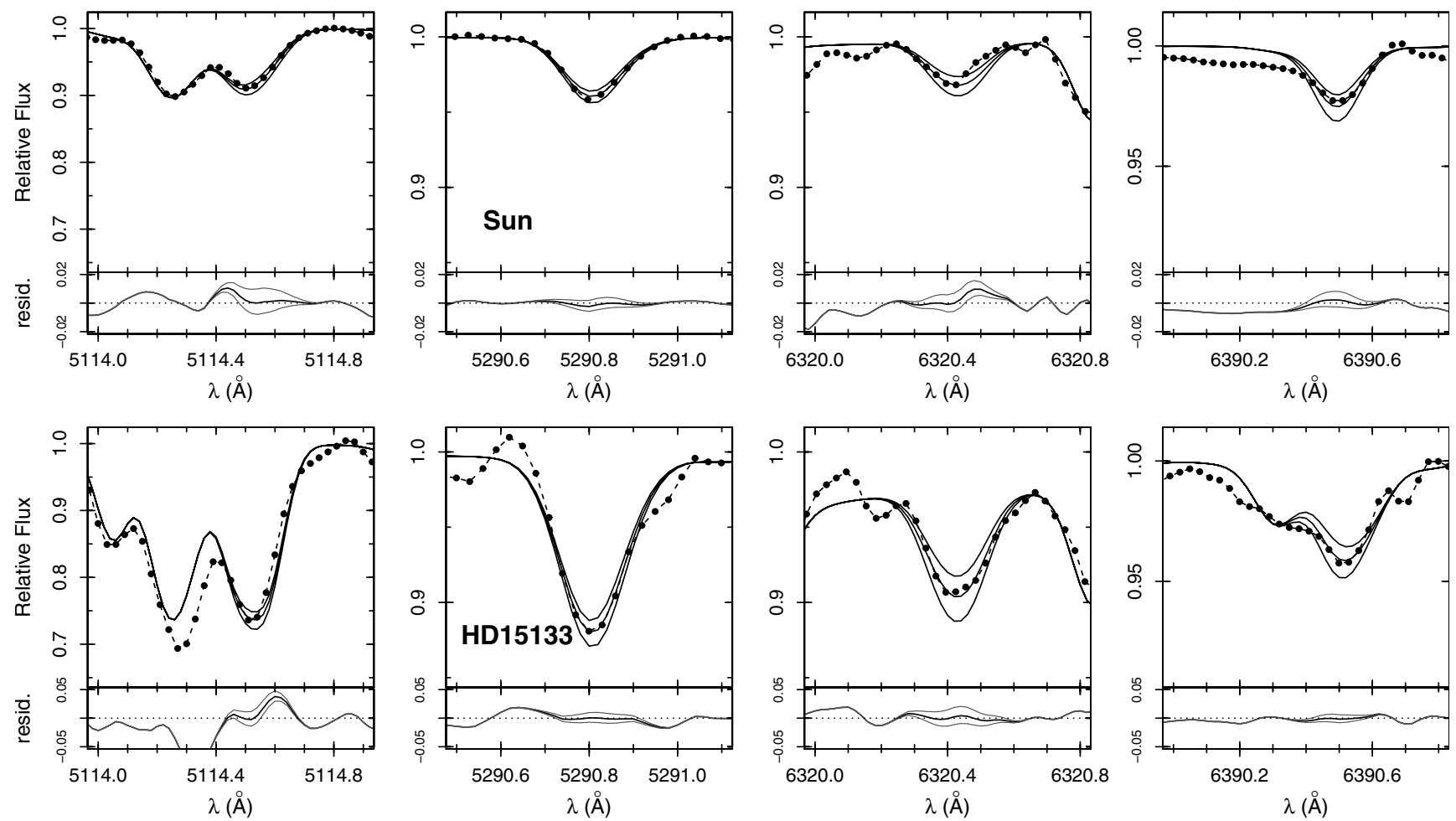

Fig. 4. Spectra in the region of La II lines. Upper panels: observed and synthetic solar spectra. Solid lines indicate the synthetic spectra for $\epsilon(\mathrm{La})=1.12,1.22,1.32$; the dots indicate the observed spectrum. Lower panels: spectrum synthesis fitting for HD 15133.

taken from Ivans et al. (2006). For lines at $5290 \AA$, $6320 \AA$ and $6390 \AA$, the HFS was obtained by using the code made available by McWilliam (1998; see Prochaska \& McWilliam 2000), as reported in Barbuy et al. (2014). The nuclide ${ }^{139} \mathrm{La}$ is the dominant isotope, contributing $99.9 \%$ to the total solar system abundance (Lawler et al. 2001a).

For the La II line at $6320 \AA$, the effect of a Ca I line at $6318.3 \AA$ that shows autoionization effects and produces a $\sim 5 \AA$ broad line was taken into account (e.g. Lecureur et al. 2007, see also the derivation of $\mathrm{Mg}$ abundances in Paper I). The Ca I autoionization line was treated by increasing its radiative broadening to reflect the much shorter lifetime of the level that is autoionized compared with the radiative lifetime of this level. The radiative broadening had to be increased by $16000 \mathrm{com}-$ pared with its standard value $\left(\propto 1 / \lambda^{2}\right.$, based on the radiative lifetimes alone) to reproduce the Ca I dip in the solar spectrum. The $\mathrm{Ca}$ abundance for each star was taken into account when computing the $\mathrm{Ca}$ I line in the $6320 \AA$ region.

\section{Europium}

Europium abundances were derived using Eu II lines at $6437 \AA$ and $6645 \AA$. We used the HFS given by Ivans et al. (2006), and isotopic proportions of $47.8 \%$ for ${ }^{151} \mathrm{Eu}$ and $52.2 \%$ for ${ }^{153} \mathrm{Eu}$ (Lawler et al. 2001b). The total $\log g f$ value was adjusted to fit the solar spectrum (Fig. 5) by adopting $\epsilon(\mathrm{Eu})=0.51$ (Grevesse \& Sauval 1998). The observed and the synthetic spectra of HD 15133 in the region of Eu II are also presented in Fig. 5.

\subsection{Spurious abundance trends}

We determined the dependence of our final abundances on $T_{\text {eff }}$. Panels a-d in Fig. 6 show the abundances vs. $T_{\text {eff }}$, and
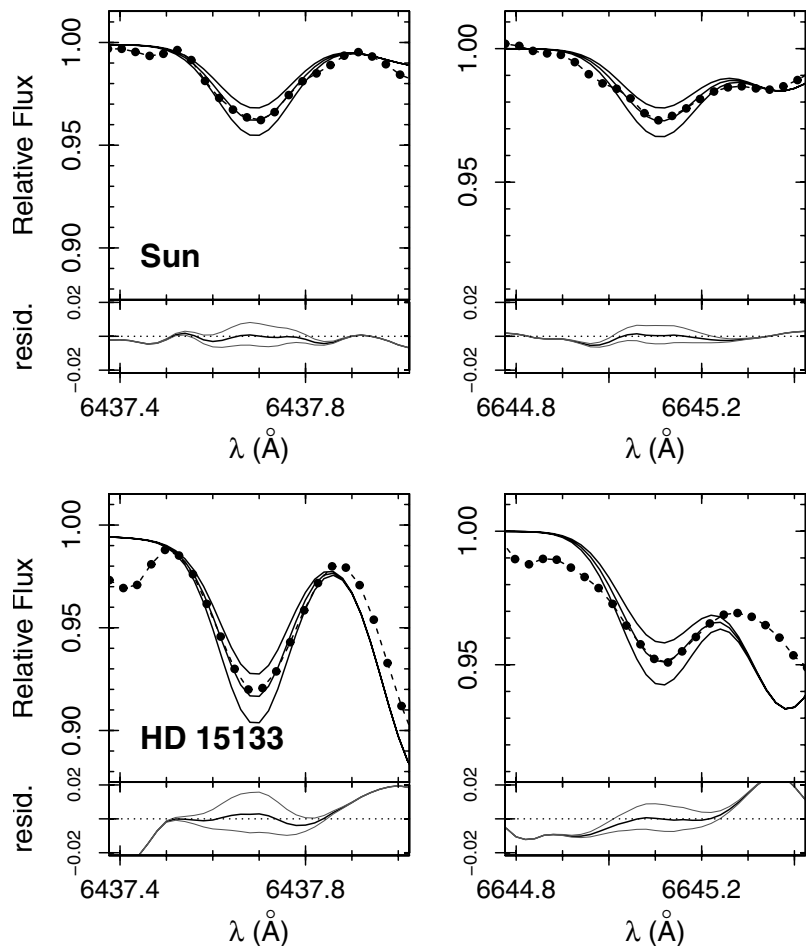

Fig. 5. Profiles of the Eu II line at $6437 \AA$ and $6645 \AA$. Upper panels: solar spectrum. Solid lines indicate the synthetic spectra for $\epsilon(\mathrm{Eu})=$ $0.41,0.51,0.61$; the dots indicate the observed spectrum. Lower panels: spectrum synthesis fitting of Eu II lines for HD 15133.

a significant trend is observed for $\mathrm{Y}, \mathrm{Ba}$, and La abundances. Following a procedure similar to that adopted in Paper I, we 


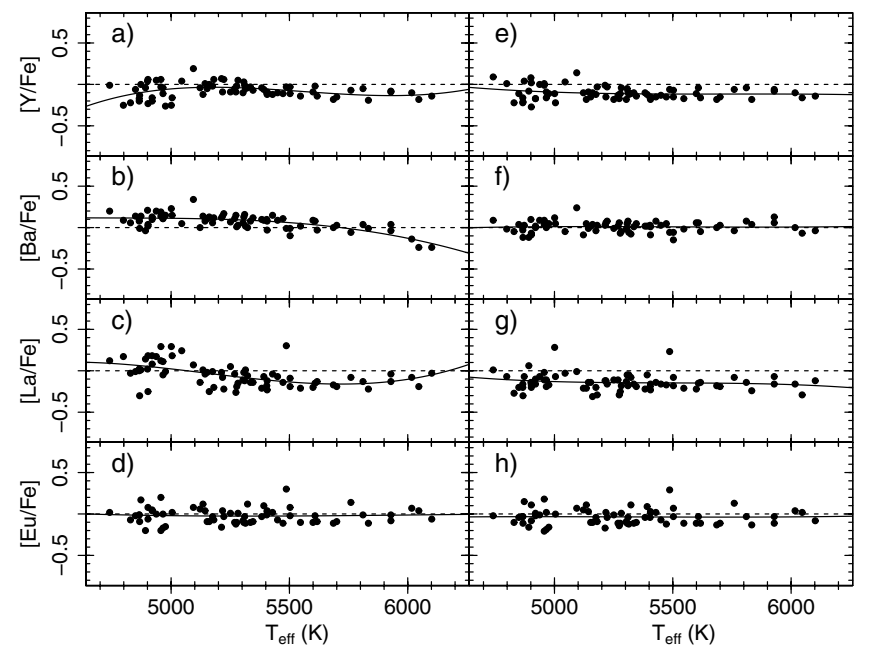

Fig. 6. Abundances of $\mathrm{Y}, \mathrm{Ba}, \mathrm{La}$, and Eu vs. temperature before (panels $a, b, c$, and $d$ ) and after (panels $e, f, g$, and $h$ ) the correction for the trend with $T_{\text {eff }}$. We considered the mean abundance in the range $5680<T_{\text {eff }}<5880 \mathrm{~K}$ as the zero point of the correction. The curve in the left panels is shown to illustrate the procedure, which was applied for abundances individually derived from each line.

corrected for this trend by fitting a second-order polynomial and by assuming that abundance trend corrections are null at $T_{\text {eff }}=5777 \mathrm{~K}$. This procedure was applied for abundances individually derived from each line, and the corrected abundances vs. $T_{\text {eff }}$ are shown in panels e-h in Fig. 6.

\subsection{Errors}

The errors in abundances result mainly from the errors of the stellar parameters and from the errors in fitting the synthetic spectra. We estimated the errors in abundances due to uncertainties in the stellar parameters, as presented in Table 3. The variations $\Delta[\mathrm{Y}, \mathrm{Ba}, \mathrm{La}, \mathrm{Eu} / \mathrm{H}]$ were computed by changing the atmospheric parameters by $\Delta T_{\mathrm{eff}}=+100 \mathrm{~K}, \Delta \log g=+0.2 \mathrm{dex}$ and $\Delta v_{\mathrm{t}}=+0.2 \mathrm{~km} \mathrm{~s}^{-1}$. This procedure was performed for three of the sample stars with different temperatures $\left(T_{\text {eff }} \sim 5900 \mathrm{~K}\right.$ and $\sim 5200 \mathrm{~K})$ and metallicities $([\mathrm{Fe} / \mathrm{H}] \sim 0.2$ and $\sim 0.5)$.

Errors in the final abundance of $\mathrm{Y}$ and $\mathrm{Eu}$ are $\sim 0.06-0.08 \mathrm{dex}$, and errors in $[\mathrm{La} / \mathrm{H}]$ are $\sim 0.10-0.12$ for the three stars. Uncertainty in the Ba abundance reach 0.19 dex for the star with high temperature, but it is smaller for cool stars ( 0.05-0.11 dex).

The final abundances of $\mathrm{Y}, \mathrm{Ba}, \mathrm{La}$ and $\mathrm{Eu}$ are given in Table 4. The uncertainties presented in this Table correspond to line-to-line scatter.

\section{Results}

The final abundances of $\mathrm{Y}, \mathrm{Ba}, \mathrm{La}$, and Eu are shown in Fig. 7, using iron as the reference element. We compare our results with data from the literature for halo stars (François et al. 2007; Ishigaki et al. 2013; Nissen \& Schuster 2011) and disk stars (Edvardsson et al. 1993; Reddy et al. 2003, 2006; Bensby et al. 2005; Nissen \& Schuster 2011; Mishenina et al. 2013). The halo data show a large scatter, as pointed out by François et al. (2007), and the trend of element-to-Fe ratio for these four elements becomes tighter above $[\mathrm{Fe} / \mathrm{H}]>-2.8$. All these samples together show the chemical evolution for these elements.
Table 3. Abundance variations with stellar parameters.

\begin{tabular}{|c|c|c|c|c|}
\hline & \multicolumn{4}{|c|}{$\begin{array}{l}\text { HD } 82943 \\
\left(T_{\text {eff }}=5929 \mathrm{~K}, \log g=4.35, v_{\mathrm{t}}=1.22 \mathrm{~km} \mathrm{~s}^{-1},[\mathrm{Fe} / \mathrm{H}]=0.23\right)\end{array}$} \\
\hline & $\begin{array}{c}\Delta T_{\text {eff }} \\
(100 \mathrm{~K})\end{array}$ & $\begin{array}{c}\Delta \log g \\
(0.2 \operatorname{dex})\end{array}$ & $\begin{array}{c}\Delta v_{\mathrm{t}} \\
\left(0.2 \mathrm{~km} \mathrm{~s}^{-1}\right) \\
\end{array}$ & Total \\
\hline$\Delta[\mathrm{Y} / \mathrm{H}]$ & +0.01 & +0.07 & -0.03 & +0.07 \\
\hline$\Delta[\mathrm{Ba} / \mathrm{H}]$ & +0.04 & +0.04 & -0.18 & +0.19 \\
\hline$\Delta[\mathrm{La} / \mathrm{H}]$ & +0.05 & +0.09 & -0.00 & +0.10 \\
\hline \multirow[t]{3}{*}{$\Delta[\mathrm{Eu} / \mathrm{H}]$} & -0.01 & +0.07 & -0.01 & +0.07 \\
\hline & \multicolumn{4}{|c|}{$\begin{array}{l}\text { HD } 87007 \\
\left(T_{\text {eff }}=5282 \mathrm{~K}, \log g=4.54, v_{\mathrm{t}}=0.61 \mathrm{~km} \mathrm{~s}^{-1},[\mathrm{Fe} / \mathrm{H}]=0.29\right)\end{array}$} \\
\hline & $\Delta T_{\text {eff }}$ & $\Delta \log g$ & $\Delta v_{\mathrm{t}}$ & Total \\
\hline$\Delta[\mathrm{Y} / \mathrm{H}]$ & +0.01 & +0.07 & -0.04 & +0.08 \\
\hline$\Delta[\mathrm{Ba} / \mathrm{H}]$ & +0.03 & +0.02 & -0.10 & +0.11 \\
\hline$\Delta[\mathrm{La} / \mathrm{H}]$ & +0.04 & +0.11 & +0.01 & +0.12 \\
\hline \multirow[t]{3}{*}{$\Delta[\mathrm{Eu} / \mathrm{H}]$} & -0.01 & +0.06 & +0.01 & +0.06 \\
\hline & \multicolumn{4}{|c|}{$\begin{array}{l}\text { HD } 93800 \\
\left(T_{\text {eff }}=5181 \mathrm{~K}, \log g=4.44, v_{\mathrm{t}}=0.30 \mathrm{~km} \mathrm{~s}^{-1},[\mathrm{Fe} / \mathrm{H}]=0.49\right)\end{array}$} \\
\hline & $\Delta T_{\text {eff }}$ & $\Delta \log g$ & $\Delta v_{\mathrm{t}}$ & Total \\
\hline$\Delta[\mathrm{Y} / \mathrm{H}]$ & +0.03 & +0.07 & -0.04 & +0.08 \\
\hline$\Delta[\mathrm{Ba} / \mathrm{H}]$ & +0.02 & +0.00 & -0.05 & +0.05 \\
\hline$\Delta[\mathrm{La} / \mathrm{H}]$ & +0.04 & +0.10 & +0.01 & +0.11 \\
\hline$\Delta[\mathrm{Eu} / \mathrm{H}]$ & -0.01 & +0.07 & -0.01 & +0.07 \\
\hline
\end{tabular}

The moderate-metallicity halo and thick-disk stars studied by Ishigaki et al. (2013) show essentially solar ratios, except for a few of them, which are enhanced in $\mathrm{La}$ and $\mathrm{Ba}$. There is also a scatter of Eu abundances and a mean overabundance of $[\mathrm{Eu} / \mathrm{Fe}]$. The halo and thick-disk data from Nissen \& Schuster (2011) are very homogeneous and close to solar. The solar-neighbourhood FGK stars from Mishenina et al. (2013) illustrate the disk abundance ratios and metallicities, which reach $[\mathrm{Fe} / \mathrm{H}] \sim+0.3$. Our sample stars are the most metal-rich sample and show the abundances of these elements for high metallicities.

\subsection{Abundance trends}

In Fig. 8 we present a more detailed view of the abundance trends within the Galactic disk by separating the sample into thin- and thick-disk components and the intermediate population. The membership assignment procedure is described in Sect. 2.1. Using the $U, V$, and $W$ space velocities from the Geneva-Copenhagen Survey (Holmberg et al. 2009; Casagrande et al. 2011), the same procedure was applied to stars studied by Edvardsson et al. (1993), Reddy et al. (2003, 2006), Bensby et al. (2005), and Mishenina et al. (2013). The assigned stellar population identifications are used to plot the literature stars with different symbols in Figs. 8-16, according to their kinematic classification in the terms employed in Paper I and as briefly described in Sect. 2.1.

The yttrium-to-iron abundance ratio decreases with increasing metallicity, which is compatible with the trend found for the disk population. $[\mathrm{Ba} / \mathrm{Fe}]$ increases with metallicities up to $[\mathrm{Fe} / \mathrm{H}] \sim-0.1$, and above this limit, the $[\mathrm{Ba} / \mathrm{Fe}]$ ratio seems to remain constant. Data from the literature indicate solar $[\mathrm{La} / \mathrm{Fe}]$ values in the metallicity range $-0.8<[\mathrm{Fe} / \mathrm{H}]<-0.3$, which decreases to $[\mathrm{La} / \mathrm{Fe}] \sim-0.1$ for stars with $[\mathrm{Fe} / \mathrm{H}] \sim 0.0$. Our sample stars folow the trend found for the literature data, which presents a decrease of La-to-Fe ratios with metallicity. The $[\mathrm{La} / \mathrm{Fe}]$ drops from $[\mathrm{La} / \mathrm{Fe}] \sim-0.1$ for stars with solar iron abundance towards $[\mathrm{La} / \mathrm{Fe}] \sim-0.2$ at high metallicities.

The literature data show a trend of Eu-to-Fe ratios dropping from $[\mathrm{Eu} / \mathrm{Fe}] \sim 0.4$ in the halo and metal-poor thick disk towards 
M. Trevisan and B. Barbuy: Heavy elements in old very metal-rich stars
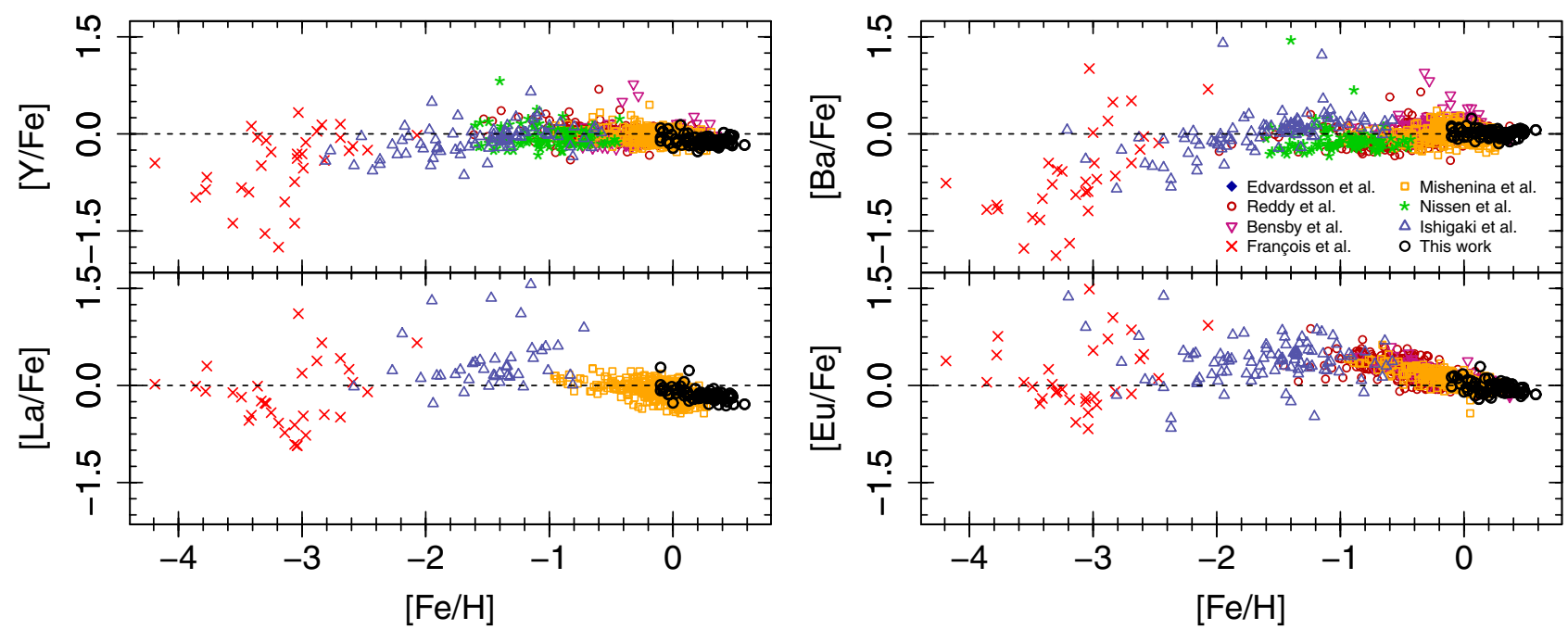

Fig. 7. Abundances of $\mathrm{Y}, \mathrm{Ba}, \mathrm{La}$ and Eu vs. metallicities. The sample stars are indicated by black open circles. Halo stars studied by François et al. (2007) and Ishigaki et al. (2013) are represented by red crosses and blue triangles, respectively. The disk stars are shown as blue diamonds (Edvardsson et al. 1993), red open circles (Reddy et al. 2003, 2006), magenta triangles Bensby et al. (2005), green stars (Nissen \& Schuster 2011) and yellow squares (Mishenina et al. 2013).
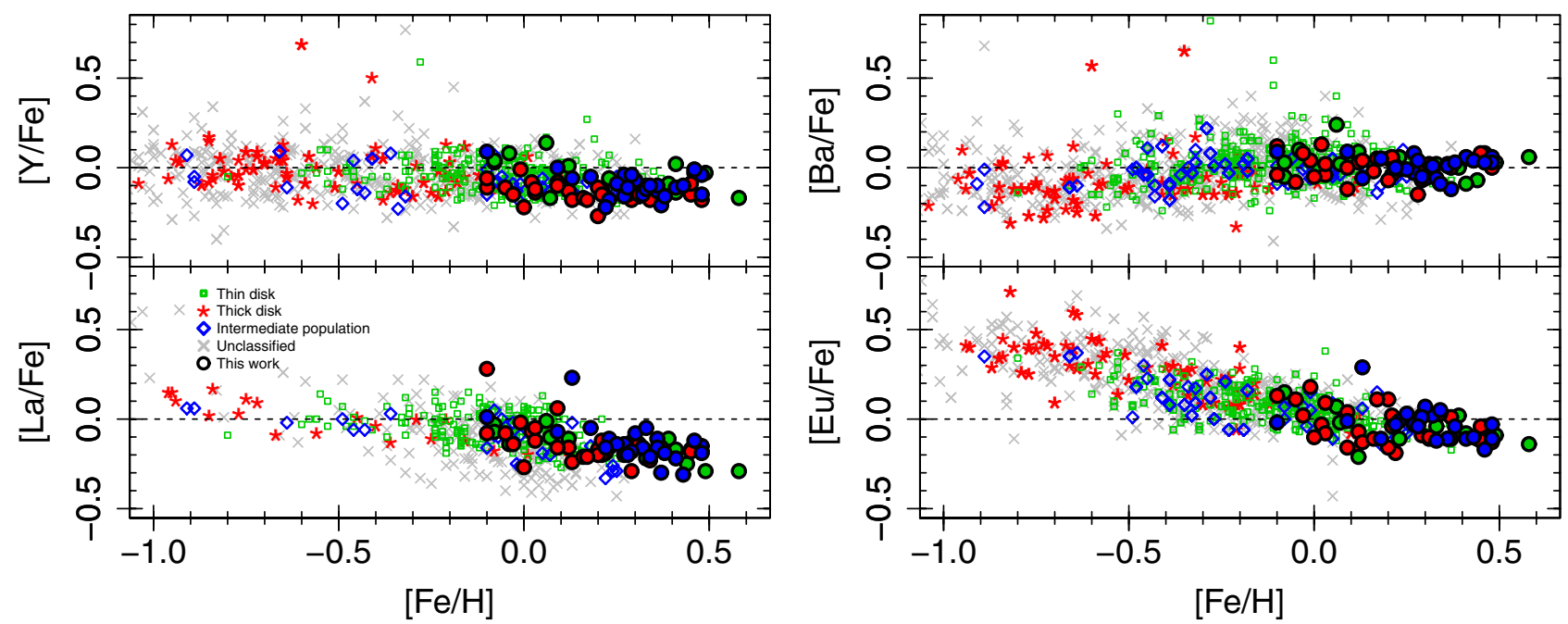

Fig. 8. Abundances of $\mathrm{Y}, \mathrm{Ba}, \mathrm{La}$, and Eu vs. metallicities. The abundances derived in this work (circles) are compared with the thin- (green squares) and thick-disk (red stars) stars and the intermediate population (blue diamonds) from Edvardsson et al. (1993), Reddy et al. (2003, 2006), Bensby et al. (2005), Nissen \& Schuster (2011), Mishenina et al. (2013), and Ishigaki et al. (2013). Grey crosses represent stars with no available $U$, $V$, and $W$ velocities, therefore no membership assignment was possible. The symbols representing our sample stars are filled according to their membership classification: thin disk (green), thick disk (red) and intermediate population (blue).

a solar value at the solar metallicity. The $[\mathrm{Eu} / \mathrm{Fe}]$ values of our sample stars follow this trend: they reach slightly sub-solar values $([\mathrm{Eu} / \mathrm{Fe}] \sim-0.1)$ at high metallicities $([\mathrm{Fe} / \mathrm{H}]>0.4)$.

\subsection{Abundance ratios among neutron-capture elements}

Abundance ratios among neutron-capture elements can provide clues on possible nucleosynthesis sites of these elements. For this purpose, we inspect the abundance ratios among Y, Ba, and La (Fig. 9), as well as the ratio between the abundances of these elements and the r-element Eu abundances (Fig. 10).

The ratio of heavy (second peak) to light (first peak) s-process elements depends on the metallicity, as discussed by
Gallino et al. (1998) and Busso et al. (1999). In Fig. 9, we show the $[\mathrm{Y} / \mathrm{Ba}],[\mathrm{Y} / \mathrm{La}]$ and $[\mathrm{Ba} / \mathrm{La}]$ vs. $[\mathrm{Fe} / \mathrm{H}]$ and vs. $[\mathrm{Ba} / \mathrm{H}]$. Stars with abundance ratios between the pure- $s$ and pure- $r$ lines should result from both processes. [Y/Ba] (Figs. 9a, b) is closer to the pure- $s$ line and tends to be compatible with the s-process production. It is slightly enhanced for stars with $[\mathrm{Fe} / \mathrm{H}] \lesssim-0.5$, which maybe requires an additional nucleosynthesis process as suggested by François et al. (2007) and Ishigaki et al. (2013), and $[\mathrm{Y} / \mathrm{Ba}] \lesssim 0.0$ for metallicities above this limit.

The Y-to-La ratios (Figs. 9c,d) are approximately constant in stars with metallicities ranging from -1.0 to +0.3 . The most metal-rich stars in our sample $([\mathrm{Fe} / \mathrm{H}]>+0.4)$ appear to have slightly higher $[\mathrm{Y} / \mathrm{La}]$ values, with values closer to the 


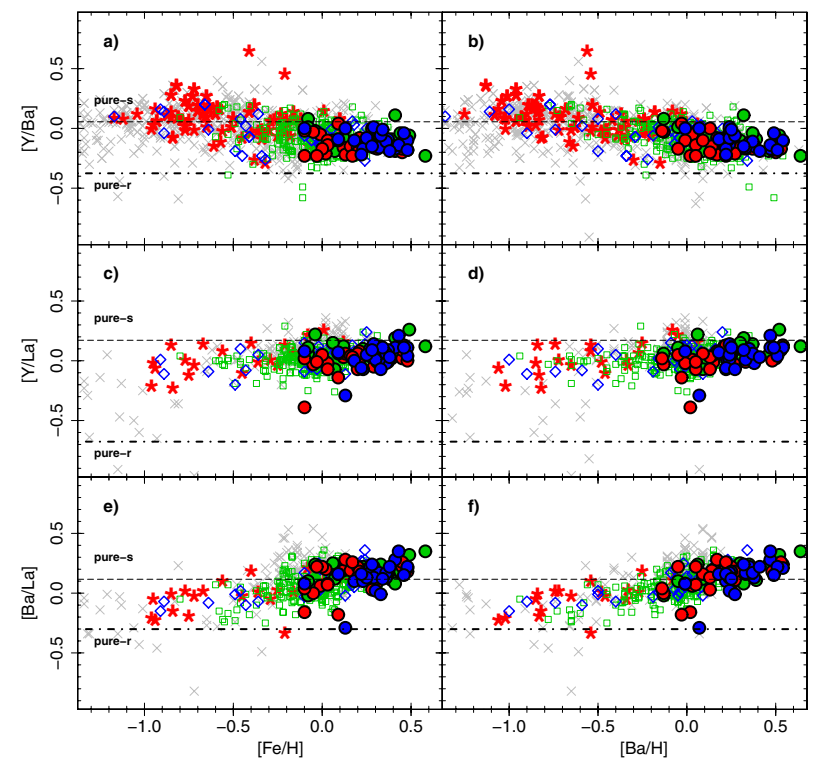

Fig. 9. Abundance ratios among neutron-capture elements. The symbols are the same as in Fig. 8. The horizontal lines indicate the abundance ratios for the $r$-process (dash-dotted) and $s$-process (dashed) components in the solar system, as predicted by Arlandini et al. (1999).

pure-s line. The $[\mathrm{Ba} / \mathrm{La}]$ values (Figs. 9e,f) increase with $[\mathrm{Fe} / \mathrm{H}]$, from $[\mathrm{Ba} / \mathrm{La}] \sim-0.1$ for metallicity -1.0 up to $[\mathrm{Ba} / \mathrm{La}] \sim+0.2$ for high metallicities.

$[\mathrm{Ba} / \mathrm{Eu}]$ vs. $[\mathrm{Fe} / \mathrm{H}]$ and vs. $[\mathrm{Eu} / \mathrm{H}]$ values (Figs. $10 \mathrm{c}, \mathrm{d}$ ) are low for thick-disk stars at low metallicities, indicating that these stars were probably enriched by SNe type II, at early times in the Galaxy. $[\mathrm{Ba} / \mathrm{Eu}]$ increases with $[\mathrm{Fe} / \mathrm{H}]$ and reaches solar values around $[\mathrm{Fe} / \mathrm{H}] \sim 0.0$. $[\mathrm{Y} / \mathrm{Eu}]$ and $[\mathrm{La} / \mathrm{Eu}]$ vs. $[\mathrm{Fe} / \mathrm{H}]$ and $[\mathrm{Eu} / \mathrm{H}]$ (Figs. 10a,b,e,f) are low for metal-poor thick disk stars, and essentially solar for most of the sample thin- and thick-disk stars and intermediate populations.

\subsection{Neutron-capture versus $\alpha$-elements}

Another interesting piece of information comes from inspecting the abundance ratios between heavy and $\alpha$-elements. Iron, which is frequently used as the reference element, is produced in both $\mathrm{SNe}$ II and SNe Ia events. The r-process component of the heavy elements and the $\alpha$-elements, in particular oxygen and magnesium, are produced in SNe II. Therefore, it is interesting to investigate how the abundance ratios of neutron-capture elements vary as a function of the abundances of oxygen and magnesium, as shown in Figs. 11 and 12.

$[\mathrm{Y} / \mathrm{O}]$ and $[\mathrm{Ba} / \mathrm{O}]$ vs. $[\mathrm{Fe} / \mathrm{H}]$ (Figs. 11a,c) increase with metallicity for all populations, but our sample stars show lower $[\mathrm{Y}, \mathrm{Ba}, \mathrm{La} / \mathrm{O}]$ with respect to the thin disk. It is interesting to note that $\mathrm{La}$ and $\mathrm{Eu}$ have similar behaviours: $[\mathrm{La} / \mathrm{O}]$ and $[\mathrm{Eu} / \mathrm{O}]$ vs. $[\mathrm{Fe} / \mathrm{H}]($ Figs. $11 \mathrm{e}, \mathrm{g})$ increase steadily with metallicity, and our results are compatible with the thin-disk behaviour. More interesting in these comparisons is the behaviour of $[\mathrm{Y} / \mathrm{O}]$ and $[\mathrm{Ba} / \mathrm{O}]$ vs. $[\mathrm{O} / \mathrm{H}]$ (Figs. 11b,d), which indicates that our sample has higher oxygen abundances than the thin-disk stars, and show higher $[\mathrm{Y}, \mathrm{Ba} / \mathrm{O}]$ ratios relative to literature thick-disk stars. The same applies to $[\mathrm{La} / \mathrm{O}]$ and $[\mathrm{Eu} / \mathrm{O}]$ vs. $[\mathrm{O} / \mathrm{H}]$ (Figs. 11f,h), but in this case, the literature thick-disk stars have the same behaviour as the sample stars.

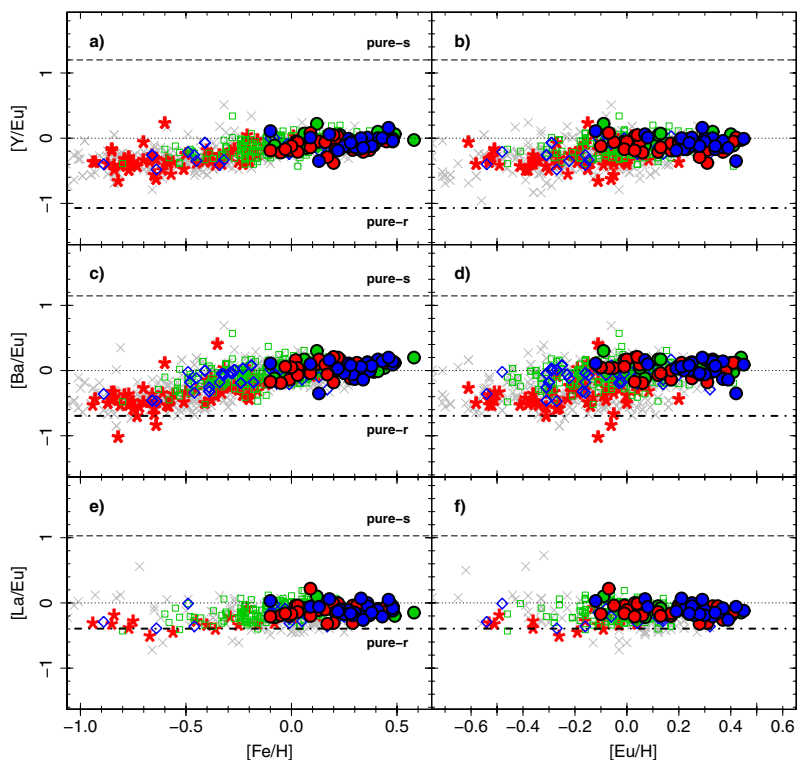

Fig. 10. Element-to-Eu ratios vs. stellar metallicities (left) and europium abundances (right). The symbols are the same as in Fig. 8. The horizontal lines indicate the abundance ratios for the $r$-process (dash-dotted) and $s$-process (dashed) components in the solar system, as predicted by Arlandini et al. (1999).

Figure 12 shows [Y, Ba, $\mathrm{La}, \mathrm{Eu} / \mathrm{Mg}]$ vs. $[\mathrm{Fe} / \mathrm{H}]$ and $[\mathrm{Mg} / \mathrm{H}]$. It is similar to Fig. 11, but it shows a strikingly better defined chemical enrichment. In addition, [Y, $\mathrm{Ba} / \mathrm{Mg}]$ ratios are compatible with the ratios in thin-disk stars. $[\mathrm{Eu} / \mathrm{Mg}]$ is constant across the whole metallicity range, indicating that $\mathrm{Eu}$ and $\mathrm{Mg}$ are produced in constant proportions in SNe type II. It is important to note that for metal-rich stars, magnesium might be a better reference element than oxygen. A plot of $[\mathrm{Mg} / \mathrm{O}]$ vs. $[\mathrm{Mg} / \mathrm{H}]$, where oxygen drops relative to magnesium at high metallicities, was given for example in Bensby et al. (2004).

\subsection{Abundances vs. Galactocentric distances}

Figures 13 and 14 show abundance ratios vs. the mean Galactocentric distances, defined as $R_{\mathrm{m}}=\left(R_{\mathrm{a}}+R_{\mathrm{p}}\right) / 2$, that is the mean of the apocentric and pericentric distances. The abundance ratios in our sample stars appear to be constant with $R_{\mathrm{m}}$. On the other hand, data from the literature indicate that $[\mathrm{Y}, \mathrm{Ba} / \mathrm{Eu}]$, [Y, $\mathrm{Ba}, \mathrm{Eu} / \mathrm{O}]$, and $\left[\mathrm{Y}, \mathrm{Ba} / \mathrm{Mg}\right.$ ] increase with $R_{\mathrm{m}}$. The observed increase of $[\mathrm{Y}, \mathrm{Ba} / \mathrm{Eu}]$ with $R_{\mathrm{m}}$ could mean that stars close to the centre are older than the outermost stars. Note that Ba has been used as an age indicator by Edvardsson et al. (1993).

$[\mathrm{Y} / \mathrm{Eu}, \mathrm{O}, \mathrm{Mg}]$ and $[\mathrm{Ba} / \mathrm{Eu}, \mathrm{O}, \mathrm{Mg}]$ (Figs. $13 \mathrm{~d}, \mathrm{e}$ and $14 a, b, e, f)$ for our thick-disk stars are clearly enhanced relative to the literature thick-disk stars. These ratios are more compatible to the literature thin-disk stars, but they are located in the inner regions, and might indicate that our sample of stars could be old inner thin-disk stars (cf. Paper I).

\subsection{Abundances vs. stellar ages}

The chemical evolution of our Galaxy with time can be inspected based on estimate of stellar ages. However, age is difficult to derive for most stars. In Paper I we derived stellar ages with uncertainties smaller than $30 \%$ for 36 sample stars. 
M. Trevisan and B. Barbuy: Heavy elements in old very metal-rich stars

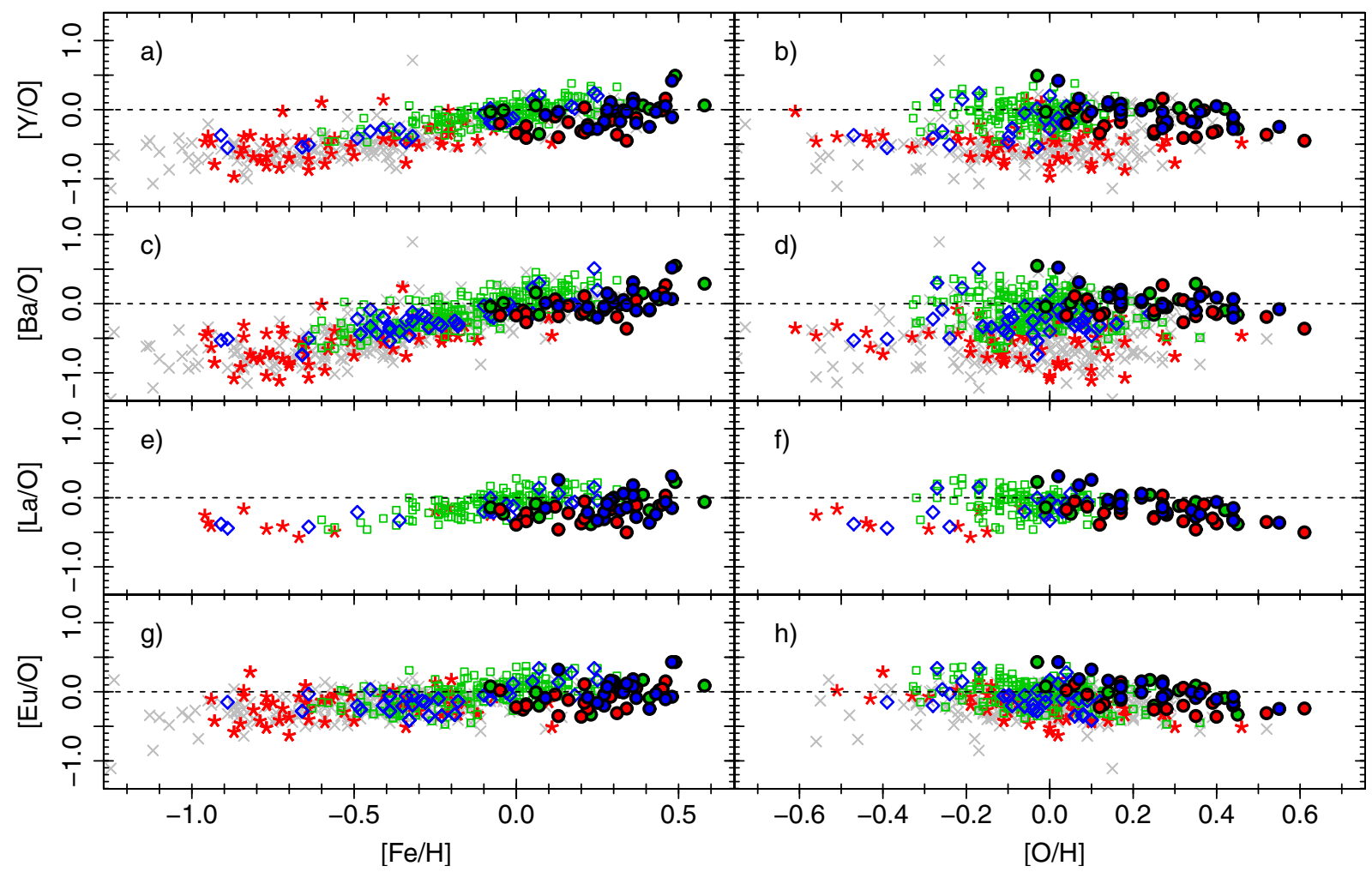

Fig. 11. Abundance ratios between neutron-capture elements and oxygen as a function of metallicity $(l e f t)$ and $[\mathrm{O} / \mathrm{H}]($ right $)$. The symbols are the same as in Fig. 8.

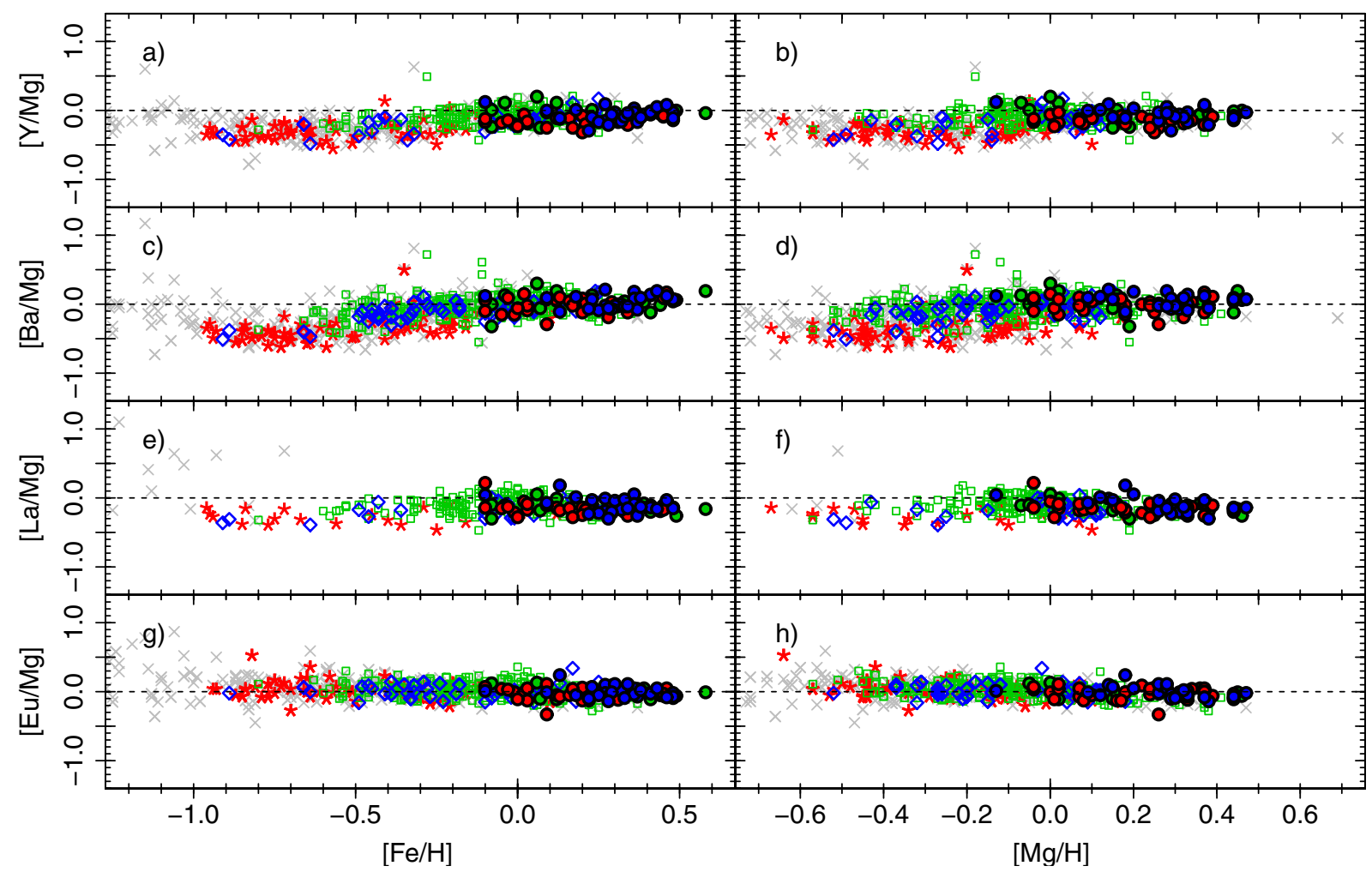

Fig. 12. Abundance ratios between neutron-capture elements and magnesium as a function of metallicity $($ left $)$ and $[\mathrm{Mg} / \mathrm{H}]($ right $)$. The symbols are the same as in Fig. 8.

Figures $15 \mathrm{a}-\mathrm{c}$ present [Y, Ba, La, Eu/O], [Y, Ba, La, Eu/Mg], $[\mathrm{Y}, \mathrm{Ba}, \mathrm{La}, \mathrm{Eu} / \mathrm{Fe}]$ vs. age, respectively. There is a clear distinction between the literature thin- and thick-disk stars in the [Y/O], $[\mathrm{Ba} / \mathrm{O}]$ vs. ages plane. Our sample matches the thin-disk 

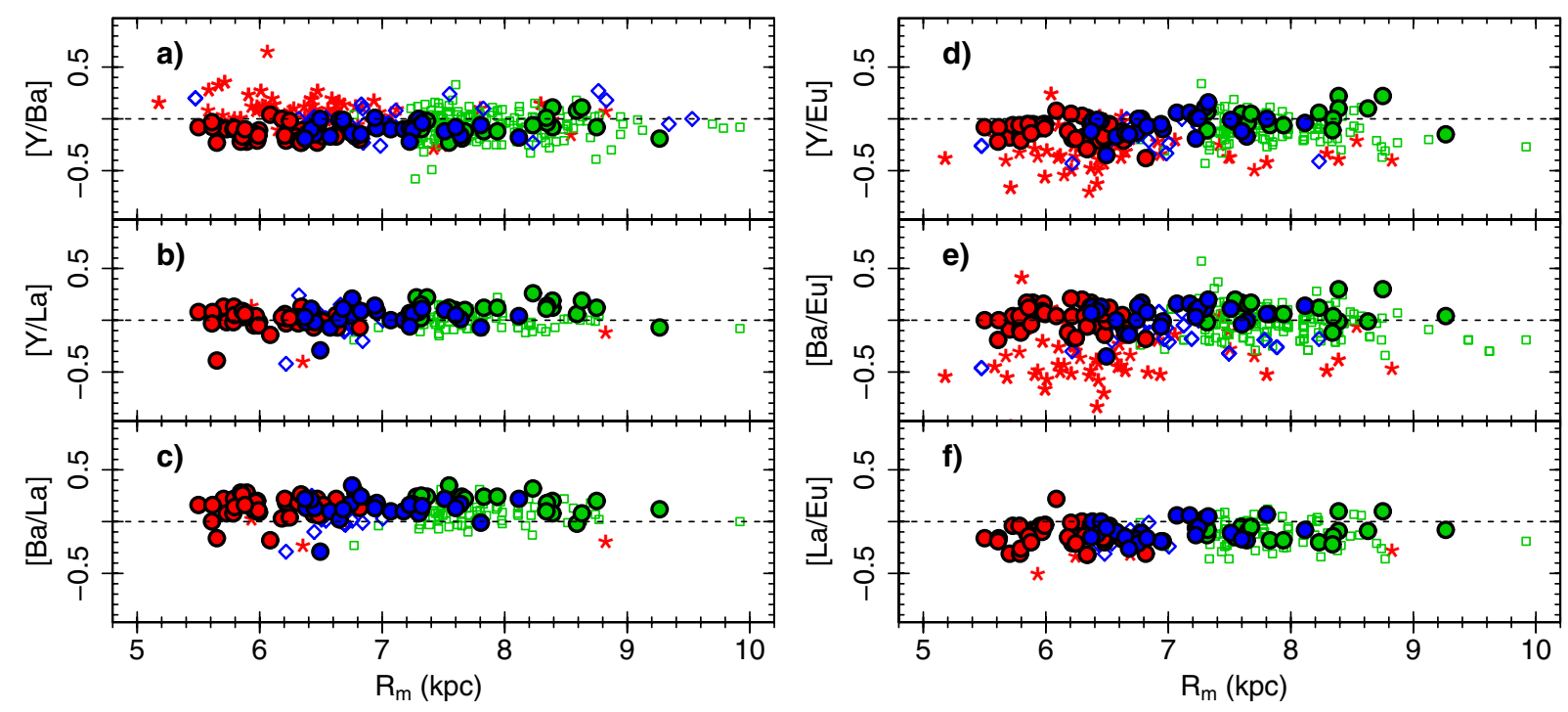

Fig. 13. Abundance ratios among neutron-capture element vs. mean Galactocentric distance. The symbols are the same as in Fig. 8.
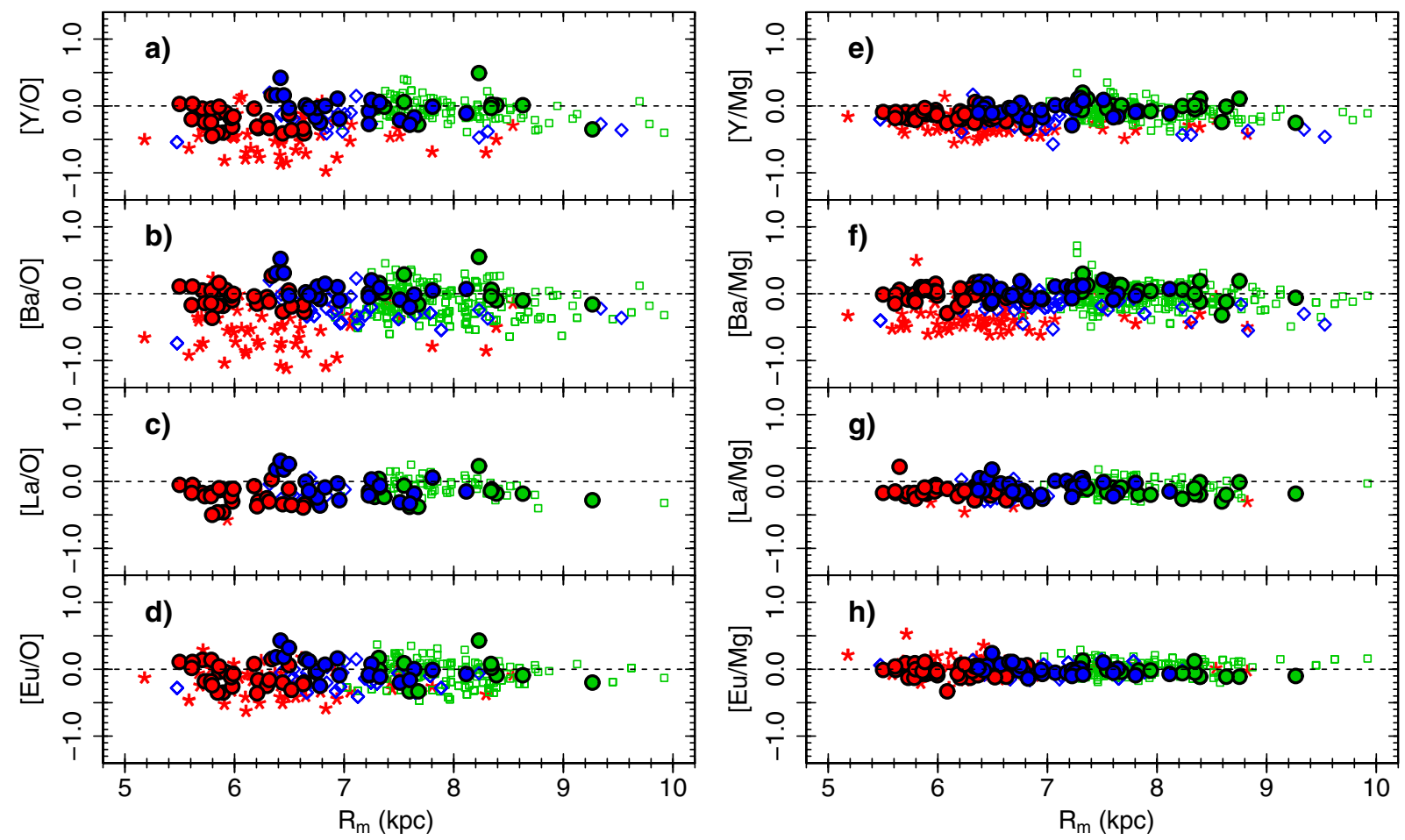

Fig. 14. [Element/O] (left panels) and [element/Mg] (right panels) ratios vs. mean Galactocentric distances. The symbols are the same as in Fig. 8.

stars, again indicating that they might be old thin-disk stars. The scatter also increases with age, and different ratios might indicate that stars were formed at different Galactocentric distances. $[\mathrm{La} / \mathrm{O}]$ is more similar to $[\mathrm{Eu} / \mathrm{O}]$, and both show solar values at all ages, except that $[\mathrm{Eu} / \mathrm{O}]$ for thick-disk stars is somewhat lower than in thin-disk stars.

Figure $15 \mathrm{~b}$ is similar to Fig. 15a, but with a smaller scatter. Abundances ratios using $\mathrm{Fe}$ as the reference element (Fig. 15c) are constant with stellar age for all populations, except $[\mathrm{Eu} / \mathrm{Fe}]$ in thick-disk stars, which increases with age. Thus the plots relative to $\mathrm{O}$ are better suited to distinguish different stellar populations. It is interesting to note that, again, the $\mathrm{Eu}$ and $\mathrm{Mg}$ production is tighly correlated for all ages.
The $[\mathrm{Ba} / \mathrm{Eu}]$ ratio, which is plotted against stellar ages in Fig. 16, is constant and close to solar across the whole age range for thin-disk and our sample stars. On the other hand, thick-disk stars show low Ba-to-Eu ratios.

\subsection{Comparison with bulge stars}

We compared the abundances of the sample stars and the bulge microlensed dwarf and subgiant stars studied by Bensby et al. (2010, 2011, 2013), and red giants from the bulge Plaut field by Johnson et al. (2012, 2013).

Figure 17 presents Y, Ba, La, and Eu abundances as a function of $[\mathrm{Fe} / \mathrm{H}]$. Literature thin- and thick-disk data are shown for 

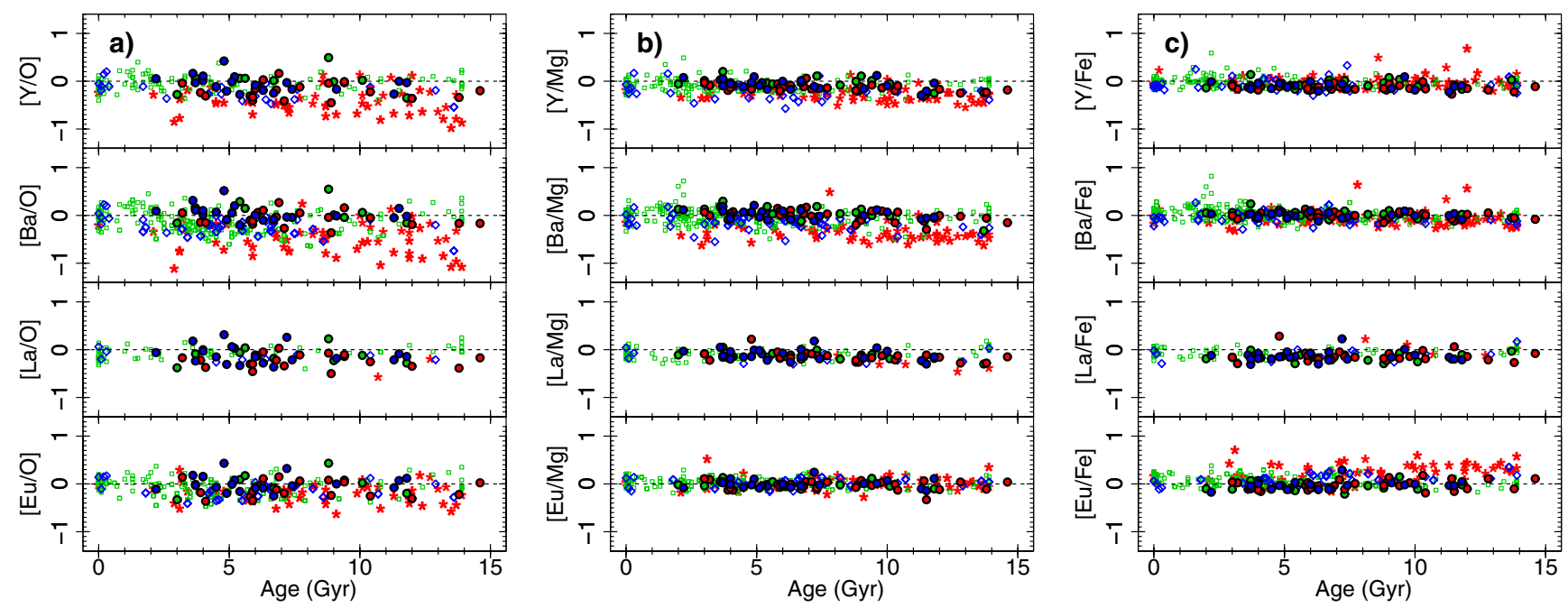

Fig. 15. Element-to-oxygen (left), element-to-magnesium (middle), and element-to-iron ratios (right) vs. stellar ages. The notation is the same as in Fig. 8. Ages for the metal-rich sample were derived as described in Paper I. Ages for samples from the literature were retrieved from the catalogue of Casagrande et al. (2011).

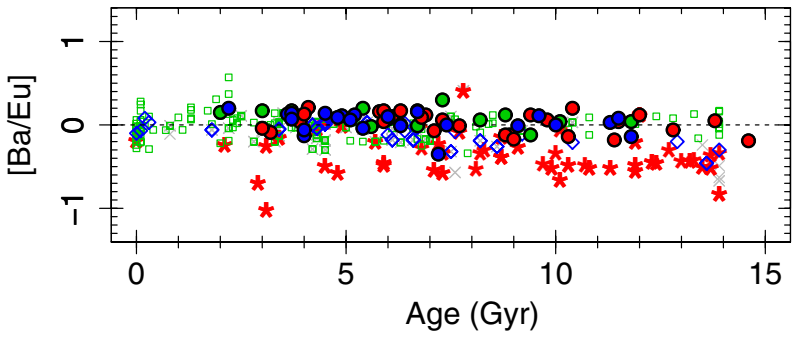

Fig. 16. $[\mathrm{Ba} / \mathrm{Eu}]$ vs. stellar ages. The notation is the same as in Fig. 8 .

comparison. There is no clear distinction between the bulge stars and our sample in terms of metallicity and [Y, Ba, Eu/Fe] abundance ratios. Figure 18 shows the abundance of $\mathrm{Y}$ and $\mathrm{Ba}$ using oxygen and magnesium as the reference element. There is a clear distinction between the trends in thin-disk and bulge stars, whereas our sample follows the trend observed for the bulge. In addition, these abundance ratios indicate that the sample stars may be the high-metallicity extension of the thick-disk stars. The trend of the thick-disk stars together with our sample is similar to the trend observed for the bulge stars.

\section{Discussion}

The mean Galactocentric distance is related to thin- and thickdisk membership probability, as can be seen in Figs. 13 and 14. The thick-disk stars have the lowest mean distances $R_{\mathrm{m}}$, since they have pericentric distances closer to the Galactic centre than thin-disk stars. For this reason, it is difficult to separate the inner thin-disk population and the thick-disk components based on kinematics alone.

Abundance ratios reflect the enrichment of the interstellar medium at the time the star was formed, and depends on the region of the Galaxy where the star formation occurred (e.g. radial gradients, that is, inner parts of the Galaxy contain stars with higher metallicities than the outer parts). If there is much radial migration in the dynamical evolution of the disk, interpreting $[X / \mathrm{Fe}]$ vs. $[\mathrm{Fe} / \mathrm{H}]$ for stars in the solar neighbourhood is difficult. However, while the kinematics of the stars may change during their lifetime, the stellar abundances do not. Therefore, chemical tagging is more efficient in identifying different stellar populations.
Our sample shows clear similarities with respect to the thindisk stars, but with a few indicators point a different nucleosynthesis for these two stellar populations. These differences are, in particular, better seen when oxygen is used as the reference element, for instance as $[\mathrm{Y} / \mathrm{O}]$ and $[\mathrm{Ba} / \mathrm{O}]$ vs. $[\mathrm{Fe} / \mathrm{H}]$ and $[\mathrm{O} / \mathrm{H}]$ (Figs. 11a-d). Our sample stars show lower [Y/O] and $[\mathrm{Ba} / \mathrm{O}]$ ratios, higher oxygen abundances, and lower $[\mathrm{Mg} / \mathrm{O}]$ than thindisk stars.

Figure 18 , which shows $[\mathrm{Y} / \mathrm{O}]$ and $[\mathrm{Ba} / \mathrm{O}]$ vs. $[\mathrm{Fe} / \mathrm{H}]$ and $[\mathrm{O} / \mathrm{H}]$, and $[\mathrm{Y} / \mathrm{Mg}]$ and $[\mathrm{Ba} / \mathrm{Mg}]$ vs. $[\mathrm{Fe} / \mathrm{H}]$ and $[\mathrm{Mg} / \mathrm{H}]$, suggests similarities between our sample and bulge stars. The lower $[\mathrm{Y} / \mathrm{O}]$ and $[\mathrm{Ba} / \mathrm{O}]$ vs. $[\mathrm{O} / \mathrm{H}]$ relative to thin-disk stars may be due to the older ages of our sample, since the contribution from AGB stars to the s-element abundances is expected to be lower in the older stars.

\section{Summary}

We have derived the abundances of the heavy elements $\mathrm{Y}, \mathrm{Ba}$, $\mathrm{La}$, and Eu for the sample of 71 metal-rich stars for which a detailed analysis was carried out in Trevisan et al. (2011).

Chemical tagging is a most efficient indicator for identifying different stellar populations. We compared our results with data from the literature for samples of thin- and thick-disk stars (Fig. 8). Our sample of stars is more metal-rich than these samples. We inspected the abundance ratios among neutron-capture elements (Figs. 9 and 10) and between heavy and $\alpha$-elements (Figs. 11 and 12). Our sample shows clear similarities with the thin-disk stars, but there are differences, in particular, the [Y/O] and $[\mathrm{Ba} / \mathrm{O}]$ vs. $[\mathrm{Fe} / \mathrm{H}]$ and $[\mathrm{O} / \mathrm{H}]$ behaviour (Figs. 11a-d).

A comparison with the bulge abundances showed that $[\mathrm{Y} / \mathrm{Fe}$, $\mathrm{O}, \mathrm{Mg}]$ and $[\mathrm{Ba} / \mathrm{Fe}, \mathrm{O}, \mathrm{Mg}]$ vs. $[\mathrm{Fe} / \mathrm{H}],[\mathrm{O} / \mathrm{H}]$, and $[\mathrm{Mg} / \mathrm{H}]$ (Figs. 17 and 18) suggest similarities between our sample and bulge stars. It would be important to be able to study more bulge stars in terms of the discriminators La, and Eu vs. the alphaelements $\mathrm{O}$ and $\mathrm{Mg}$, and compare them with our sample to verify the degree of similarity between this sample and bulge stars.

The similar behaviour of $\mathrm{La}$ and $\mathrm{Eu}$, which seems to vary in lockstep, as well as the constant value of $[\mathrm{Eu} / \mathrm{Mg}]$ vs. $[\mathrm{Mg} / \mathrm{H}]$ is remarkable. It suggests that $\mathrm{Eu}$ and $\mathrm{Mg}$ are produced in the same $\mathrm{SNe}$ type II, in the same proportions, at least at metallicities $[\mathrm{Fe} / \mathrm{H}]>-1$. 


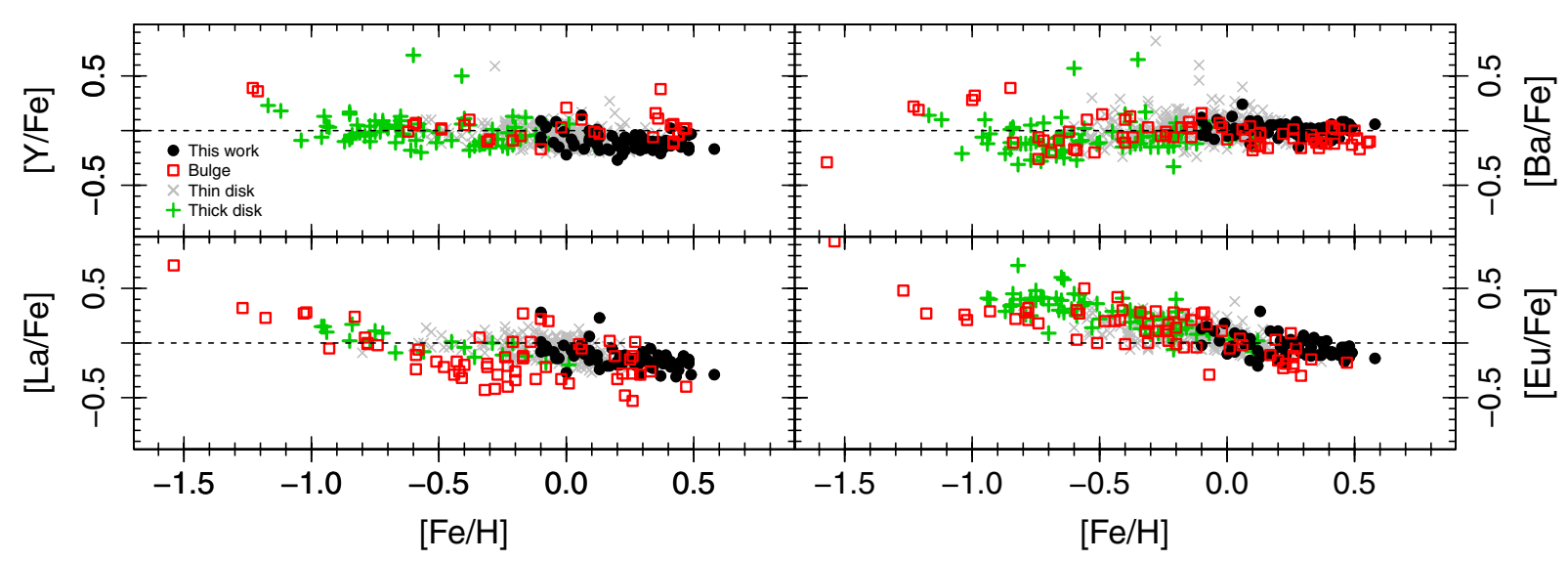

Fig. 17. Abundances of $\mathrm{Y}, \mathrm{Ba}, \mathrm{La}$, and Eu vs. $[\mathrm{Fe} / \mathrm{H}]$ for the sample stars (black dots) and bulge stars (red squares). The abundances of the bulge population were derived by Bensby et al. (2010, 2011, 2013, microlensed dwarf stars) and by Johnson et al. (2012, RGB stars). The grey and green crosses indicate thin- and thick-disk stars.
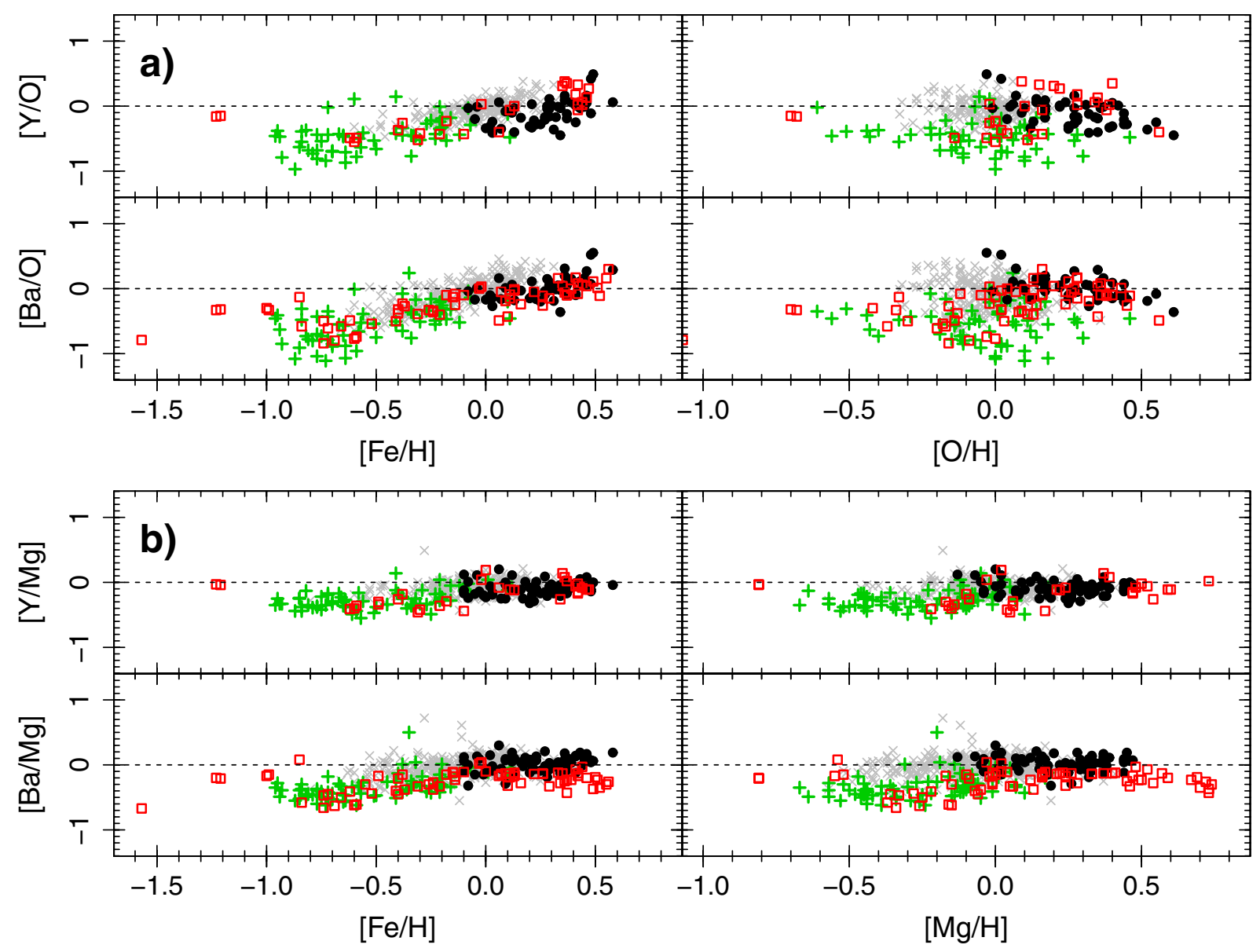

Fig. 18. Abundance ratios between neutron-capture elements and $\alpha$-elements for the sample stars and for bulge stars. Upper panel: [Y/O] and $[\mathrm{Ba} / \mathrm{O}]$ abundance ratios vs. $[\mathrm{Fe} / \mathrm{H}]($ left $)$ and $[\mathrm{O} / \mathrm{H}]$ (right). Lower panel: $[\mathrm{Y} / \mathrm{Mg}]$ and $[\mathrm{Ba} / \mathrm{Mg}]$ abundance ratios vs. $[\mathrm{Fe} / \mathrm{H}]($ left $)$ and $[\mathrm{Mg} / \mathrm{H}]$ (right). The notation is the same as in Fig. 17.

In conclusion, our results indicate that the sample stars might either be bulge stars, which justifies the denomination "bulgelike", as previously suggested by Pompéia et al. (2003), or inner old thin-disk stars (Haywood 2008). In either cases they would have been ejected by the bar towards the solar neighbourhood. It is less likely that they represent a high-metallicity complement to the thick-disk stars. Finally, since some abundance ratios are different from those in the bulge and thin- and thick-disk stars, the sample stars may be yet another stellar population. This makes additional verifications desirable.

Acknowledgements. We would like to thank the anonymous referee for his or her helpful comments, which helped to improve this manuscript. M.T. acknowledges the support of FAPESP, process No. 2012/05142-5. The observations were carried out within Brazilian time in a ESO-ON agreement, and within an IAGON agreement funded by FAPESP project No. 1998/10138-8. We acknowledge partial financial support from CNPq and Fapesp. 
M. Trevisan and B. Barbuy: Heavy elements in old very metal-rich stars

\section{References}

Allende Prieto, C., Barklem, P. S., Lambert, D. L., \& Cunha, K. 2004, A\&A, 420, 183

Arlandini, C., Käppeler, F., Wisshak, K., et al. 1999, ApJ, 525, 886

Asplund, M., Grevesse, N., Sauval, A. J., \& Scott, P. 2009, ARA\&A, 47, 481

Barbuy, B., Perrin, M.-N., Katz, D., et al. 2003, A\&A, 404, 661

Barbuy, B., Chiappini, C., Cantelli, E., et al. 2014, A\&A, in press, DOI: 10.1051/0004-6361/201424311

Bedin, L. R., Piotto, G., Carraro, G., King, I. R., \& Anderson, J. 2006, A\&A, 460, L27

Bensby, T., Feltzing, S., \& Lundström, I. 2004, A\&A, 415, 155

Bensby, T., Feltzing, S., Lundström, I., \& Ilyin, I. 2005, A\&A, 433, 185

Bensby, T., Feltzing, S., Johnson, J. A., et al. 2010, A\&A, 512, A41

Bensby, T., Adén, D., Meléndez, J., et al. 2011, A\&A, 533, A134

Bensby, T., Yee, J. C., Feltzing, S., et al. 2013, A\&A, 549, A147

Busso, M., Gallino, R., \& Wasserburg, G. J. 1999, ARA\&A, 37, 239

Carraro, G., Girardi, L., \& Marigo, P. 2002, MNRAS, 332, 705

Casagrande, L., Ramírez, I., Meléndez, J., Bessell, M., \& Asplund, M. 2010, A\&A, 512, A54

Casagrande, L., Schoenrich, R., Asplund, M., et al. 2011, A\&A, 530, A138

Cayrel, R., Perrin, M.-N., Barbuy, B., \& Buser, R. 1991, A\&A, 247, 108

Cescutti, G., \& Chiappini, C. 2014, A\&A, 565, A51

Chiappini, C., Frischknecht, U., Meynet, G., et al. 2011, 474, 666

Coelho, P., Barbuy, B., Meléndez, J., Schiavon, R. P., \& Castilho, B. V. 2005, A\&A, 443, 735

Demarque, P., Woo, J., Kim, Y., \& Yi, S. K. 2004, ApJS, 155, 667

Edvardsson, B., Andersen, J., Gustafsson, B., et al. 1993, A\&A, 275, 101

François, P., Depagne, E., Hill, V., et al. 2007, A\&A, 476, 935

Gallino, R., Arlandini, C., Busso, M., et al. 1998, ApJ, 497, 388

Grenon, M. 1999, Ap\&SS, 265, 331

Grevesse, N., \& Sauval, A. J. 1998, SSRv, 85, 161

Grevesse, N., Scott, P., Asplund, M., \& Sauval, A. J. 2014, A\&A, in press, DOI: $10.1051 / 0004-6361 / 201424111$

Gustafsson, B., Edvardsson, B., Eriksson, K., et al. 2008, A\&A, 486, 951

Hannaford, P., Lowe, R. M., Grevesse, N., Biemont, E., \& Whaling, W. 1982, ApJ, 261, 736

Haywood, M. 2008, MNRAS, 388, 1175

Hill, V., Plez, B., Cayrel, R., et al. 2002, A\&A, 387, 560

Holmberg, J., Nordström, B., \& Andersen, J. 2009, A\&A, 501, 941

Ishigaki, M. N., Aoki, W., \& Chiba, M. 2013, ApJ, 771, 67

Ivans, I. I., Simmerer, J., Sneden, C., et al. 2006, ApJ, 645, 613

Jacobson, H. R., \& Friel, E. D. 2013, AJ, 145, 107

Johnson, C. I., Rich, R. M., Kobayashi, C., \& Fulbright, J. P. 2012, ApJ, 749, 175
Johnson, C. I., McWilliam, A., \& Rich, R. M. 2013, ApJ, 775, L27

Käppeler, F., Beer, H., \& Wisshak, K. 1989, Rep. Prog. Phys., 52, 945

Käppeler, F., Gallino, R., Bisterzo, S., \& Aoki, W. 2011, Rev. Mod. Phys., 83, 157

Kaufer, A., Stahl, O., Tubbesing, S., et al. 2000, in SPIE Conf. 4008, eds. M. Iye, \& A. F. Moorwood, 459

Kratz, K.-L., Farouqi, K., Pfeiffer, B., et al. 2007, ApJ, 662, 39

Lawler, J. E., Bonvallet, G., \& Sneden, C. 2001a, ApJ, 556, 452

Lawler, J. E., Wickliffe, M. E., den Hartog, E. A., \& Sneden, C. 2001b, ApJ, 563,1075

Lebzelter, T., Uttenthaler, S., Straniero, O., \& Aringer, B. 2013, A\&A, 554, A 30

Lecureur, A., Hill, V., Zoccali, M., et al. 2007, A\&A, 465, 799

Lodders, K., Palme, H., \& Gail, H.-P. 2009, Landolt Börnstein, 44

Luyten, W. J. 1980, New Luyten Catalogue of Stars with Proper Motions Larger than Two Tenths of an Arcsecond (Minneapolis: University of Minnesota)

McWilliam, A. 1998, AJ, 115, 1640

Mishenina, T. V., Pignatari, M., Korotin, S. A., et al. 2013, A\&A, 552, A128

Nissen, P. E., \& Schuster, W. J. 2011, A\&A, 530, A15

Pompéia, L., Barbuy, B., \& Grenon, M. 2003, ApJ, 592, 1173

Prochaska, J. X., \& McWilliam, A. 2000, ApJ, 537, L57

Raboud, D., Grenon, M., Martinet, L., Fux, R., \& Udry, S. 1998, A\&A, 335, L61

Reddy, B. E., Tomkin, J., Lambert, D. L., \& Allende Prieto, C. 2003, MNRAS, 340,304

Reddy, B. E., Lambert, D. L., \& Allende Prieto, C. 2006, MNRAS, 367, 1329

Rosswog, S., Korobkin, O., Arcones, A., Thielemann, F.-K., \& Piran, T. 2014, MNRAS, 439, 744

Schatz, H., Aprahamian, A., Barnard, V., et al. 2001, Phys. Rev. Lett., 86, 3471

Seeger, P. A., Fowler, W. A., \& Clayton, D. D. 1965, ApJS, 11, 121

Simmerer, J., Sneden, C., Cowan, J. J., et al. 2004, ApJ, 617, 1091

Siqueira-Mello, C., \& Barbuy, B. 2014, Ap\&SS, in press

Siqueira-Mello, C., Hill, V., Barbuy, B., et al. 2014, A\&A, 565, A93

Sneden, C., Cowan, J. J., \& Gallino, R. 2008, ARA\&A, 46, 241

Soubiran, C., Bienaymé, O., \& Siebert, A. 2003, A\&A, 398, 141

Soubiran, C., Le Campion, J., Cayrel de Strobel, G., \& Caillo, A. 2010, A\&A, 515, A 111

Spite, M., Huille, S., Spite, F., \& Francois, P. 1987, A\&AS, 71, 591

Thielemann, F.-K., Arcones, A., Käppeli, R., et al. 2011, Prog. Part. Nucl. Phys., 66,346

Travaglio, C., Gallino, R., Arnone, E., et al. 2004, ApJ, 601, 864

Trevisan, M., Barbuy, B., Eriksson, K., et al. 2011, A\&A, 535, A42

Truran, J. W. 1981, A\&A, 97, 391

Wanajo, S., \& Ishimaru, Y. 2006, Nucl. Phys. A, 777, 676

Wanajo, S., Sekiguchi, Y., Nishimura, N., et al. 2014, ApJ, 789, L39 